\title{
The Network Origins of Aggregate Fluctuations*
}

\author{
Daron Acemoglu ${ }^{\dagger}$ Vasco M. Carvalho ${ }^{\ddagger} \quad$ Asuman Ozdaglar ${ }^{\S} \quad$ Alireza Tahbaz-Salehi ${ }^{\llbracket}$
}

October 2011

\begin{abstract}
This paper argues that in the presence of intersectoral input-output linkages, microeconomic idiosyncratic shocks may lead to aggregate fluctuations. In particular, it shows that, as the economy becomes more disaggregated, the rate at which aggregate volatility decays is determined by the structure of the network capturing such linkages. Our main results provide a characterization of this relationship in terms of the importance of different sectors as suppliers to their immediate customers as well as their role as indirect suppliers to chains of downstream sectors. Such higher-order interconnections capture the possibility of "cascade effects" whereby productivity shocks to a sector propagate not only to its immediate downstream customers, but also indirectly to the rest of the economy. Our results highlight that sizable aggregate volatility is obtained from sectoral idiosyncratic shocks only if there exists significant asymmetry in the roles that sectors play as suppliers to others, and that the "sparseness" of the input-output matrix is unrelated to the nature of aggregate fluctuations.
\end{abstract}

Keywords: Business cycle, aggregate volatility, diversification, input-output linkages, intersectoral network, cascades.

JEL Classification: C67, D57, E32

*This paper combines material from Carvalho's Ph.D. dissertation at the University of Chicago (Carvalho, 2008) and Acemoglu, Ozdaglar, and Tahbaz-Salehi (2010). We thank the editor, Stephen Morris, and four anonymous referees for very helpful remarks and suggestions. We thank Stefana Stantcheva for excellent research assistance and Bill Kerr and Kaushik Ghosh for initial help with the NBER manufacturing productivity database. Carvalho thanks his advisor Lars Hansen and other members of his thesis committee, Robert Lucas and Timothy Conley. We are also grateful to John Fernald, Xavier Gabaix, Ali Jadbabaie, Alp Simsek, Hugo Sonnenschein, Jean Tirole, Jaume Ventura and numerous seminar and conference participants for useful feedback and suggestions. Carvalho acknowledges financial support from the Government of Catalonia (grant 2009SGR1157), the Spanish Ministry of Education and Science (grants Juan de la Cierva, JCI2009-04127, ECO2008-01665 and CSD2006-00016) and Barcelona GSE Research Network. Acemoglu, Ozdaglar and Tahbaz-Salehi acknowledge financial support from the Toulouse Network of Information Technology, National Science Foundation (Grant 0735956) and the Air Force Office of Scientific Research (Grant FA9550-09-1-0420).

${ }^{\dagger}$ Department of Economics, Massachusetts Institute of Technology.

${ }^{\ddagger}$ CREI and University of Pompeu Fabra.

${ }^{\S}$ Department of Electrical Engineering and Computer Science, Massachusetts Institute of Technology.

[Decisions, Risk, and Operations Division, Columbia Business School, Columbia University. 


\section{Introduction}

The possibility that significant aggregate fluctuations may originate from microeconomic shocks to firms or disaggregated sectors has long been discarded in macroeconomics due to a "diversification argument". As argued by Lucas (1977), among others, such microeconomic shocks would average out and thus, would only have negligible aggregate effects. In particular, the argument goes, aggregate output concentrates around its mean at a very rapid rate: in an economy consisting of $n$ sectors hit by independent shocks, aggregate fluctuations would have a magnitude proportional to $1 / \sqrt{n}$ - a negligible effect at high levels of disaggregation.

This argument, however, ignores the presence of interconnections between different firms and sectors, functioning as a potential propagation mechanism of idiosyncratic shocks throughout the economy. The possible role of such interconnections in propagation of shocks was highlighted during the debate leading to the recent auto industry bailout. Appearing before the Senate Banking Committee in November 2008, Alan R. Mulally, the chief executive of Ford, requested emergency government support for General Motors and Chrysler, Ford's traditional rivals. Mulally argued that given the significant overlap in the suppliers and dealers of the three automakers, the collapse of either company could unleash a disastrous chain of events for his company as well as the entire economy (Mulally, 2008). The possibility of such "cascade effects" due to interconnections was also a key argument in government bailouts of several large financial institutions during the financial crisis of 2007-2009.

This paper shows that the types of interconnections emphasized by Mulally indeed imply that the effects of microeconomic shocks may not remain confined to where they originate. Rather, such shocks may propagate throughout the economy, affect the output of other sectors, and generate sizable aggregate effects. Our main contribution is to provide a general mathematical framework for the analysis of such propagations and characterize how the extent of propagations of idiosyncratic shocks and their role in aggregate fluctuations depend on the structure of interactions between different sectors.

The following simple example illustrates the standard diversification argument and why it may not apply in the presence of interconnections.

Example 1. Consider the economy depicted in Figure 1(a) consisting of $n$ non-interacting sectors. As $n$ increases and the economy becomes more disaggregated, the diversification argument based on the law of large numbers implies that independent sectoral shocks will average out rapidly at the rate $\sqrt{n}$. An identical reasoning is applicable to the economy depicted in Figure 1(b), where each sector relies equally on the outputs of all other sectors. The symmetric structure of this economy ensures that aggregate output is a symmetric function of the shocks to each sector, implying that the diversification argument applies.

Such an argument would not be valid, however, if intersectoral input-output linkages exhibit 


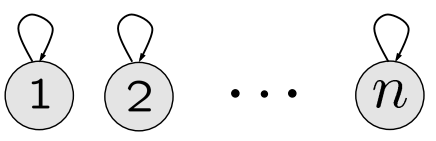

(a) An economy in which no sector relies on other sectors for production

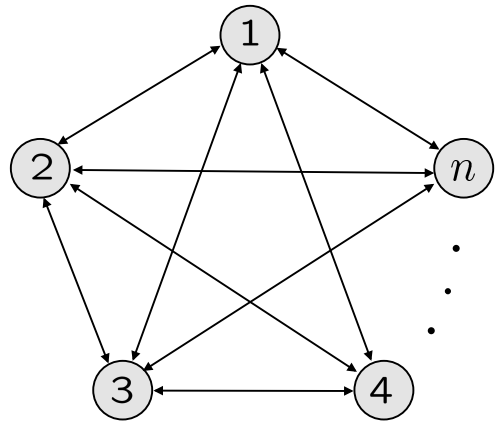

(b) An economy in which each sector relies equally on all other sectors

Figure 1: The network representations of two symmetric economies

no such symmetries. For instance, consider the economy depicted in Figure 2, in which sector 1 is the sole input supplier to all others. In this case, as $n$ increases, sectoral shocks do not average out: even when $n$ is large, shocks to sector 1 propagate strongly to the rest of the economy, generating significant aggregate fluctuations.

Even though the "star network" in Figure 2 illustrates that, in the presence of interconnections, sectoral shocks may not average out, it is also to some extent an extreme example. A key question, therefore, is whether the effects of micro shocks can be ignored in economies with more realistic patterns of interconnections. The answer naturally depends on whether the intersectoral network structures of actual economies resemble the economies in Figure 1 or the star network structure in Figure 2. Figure 3 gives a first glimpse of the answer by depicting the input-output linkages between 474 U.S. industries in 1997. It suggests that even though the pattern of sectoral interconnections is not represented by a star network, it is also significantly different from the networks depicted in Figure 1. In fact, as our analysis in Section 4 will show, in many ways the structure of the intersectoral input-output relations of the U.S. economy is much more similar to that of Figure 2, as a small number of sectors play a disproportionately important role as input suppliers to

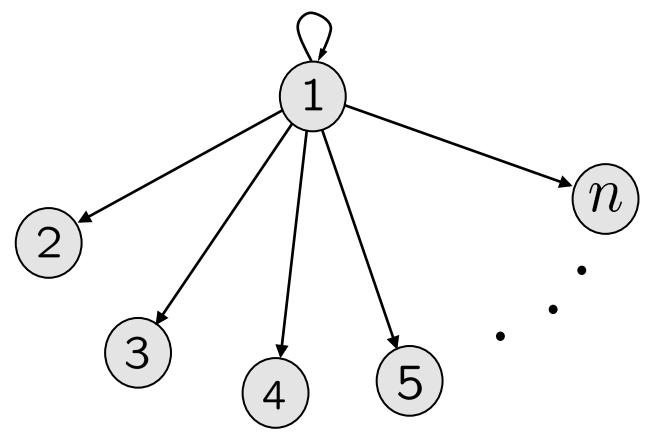

Figure 2: An economy where one sector is the only supplier of all other sectors. 


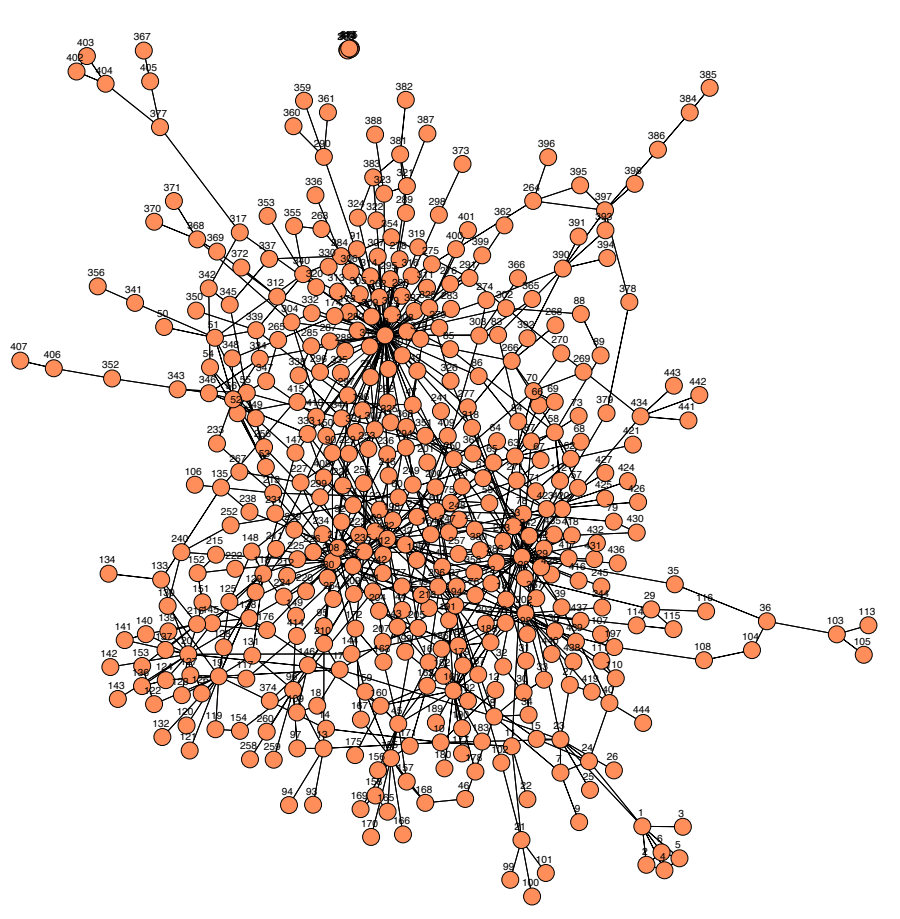

Figure 3: Intersectoral network corresponding to the U.S. input-output matrix in 1997 (Source: Bureau of Economic Analysis. See Section 4 for more details on the data). Each vertex corresponds to a sector in the 1997 benchmark detailed commodity-by-commodity direct requirements table. For every input transaction above $5 \%$ of the total input purchases of the destination sector, a link between two vertices is drawn.

others. Consequently, the interplay of sectoral shocks and the intersectoral network structure may generate sizable aggregate fluctuations.

In order to develop these ideas more systematically, we consider a sequence of economies $\left\{\mathcal{E}_{n}\right\}_{n \in \mathbb{N}}$, corresponding to different levels of disaggregation. ${ }^{1}$ Each economy $\mathcal{E}_{n}$ consists of $n$ sectors whose input requirements are captured by an $n \times n$ matrix $W_{n}$. Entry $(i, j)$ of this matrix captures the share of sector $j$ 's product in sector $i$ 's production technology. Its $j$-th column sum, which we refer to as the degree of sector $j$, corresponds to the share of $j$ 's output in the input supply of the entire economy. Given the sequence of economies $\left\{\mathcal{E}_{n}\right\}_{n \in \mathbb{N}}$, we investigate whether aggregate volatility, defined as the standard deviation of $\log$ output, vanishes as $n \rightarrow \infty$. We show that in certain cases, such as the star network, the law of large numbers fails and aggregate output does not concentrate around a constant value.

The main focus of our analysis, however, is on the more interesting cases in which the law of large numbers holds, yet the structure of the intersectoral network still has a defining effect on aggregate fluctuations. We show that sectoral interconnections may imply that aggregate output concentrates around its mean at a rate significantly slower than $\sqrt{n}$. Such slower rates of decay mean that sectoral shocks would have a more significant role in creating aggregate fluctuations, even at high levels of disaggregation. Our results also establish that slow rates of decay of ag-

\footnotetext{
${ }^{1}$ In our model economy, the total supply of labor is fixed. Therefore, an increase in the number of sectors is equivalent to an increase in the level of disaggregation of the economy.
} 
gregate volatility may have two related but distinct causes. First, they may be due to first-order interconnections: shocks to a sector which is a supplier to a disproportionally large number of other sectors propagate directly to those sectors. Second, they may be due to higher-order interconnections: low productivity in one sector leads to a reduction in production of not only its immediate downstream sectors but also a sequence of sectors interconnected to one another, creating cascade effects.

In addition to illustrating the role of interconnections in creating aggregate fluctuations from sectoral shocks, we prove three key theorems characterizing the rate of convergence of aggregate volatility, and hence quantifying the impact of interconnections, in terms of the structural properties of the intersectoral network. Theorem 2 provides a lower bound in terms of the extent of asymmetry across sectors captured by variations in their degrees. It shows that higher variations in the degrees of different sectors imply lower rates of decay for aggregate volatility. A corollary to this result shows that if the empirical distribution of degrees of the intersectoral network can be approximated by a power law (Pareto distribution) with shape parameter $\beta \in(1,2)$, then aggregate volatility decays at a rate slower than $n^{(\beta-1) / \beta}$. Theorem 3 provides tighter lower bounds in terms of a measure of second-order interconnectivity between different sectors. This characterization is important because two economies with identical empirical degree distributions (first-order connections) may have significantly different levels of aggregate volatility resulting from the roles that some sectors play as indirect input suppliers to the economy through chains of downstream sectors. We use this extended characterization to provide a bound in terms of the empirical distribution of the second-order degrees of different sectors within the economy, where the second-order degree of sector $i$ is defined as the weighted sum of the degrees of sectors that demand inputs from $i$ with weights given by the input share of $i$ in the production of these sectors. In particular, we show that if the empirical distribution of the second-order degrees can be approximated by a power law with shape parameter $\zeta \in(1,2)$, then aggregate volatility decays at a rate slower than $n^{(\zeta-1) / \zeta}$. Finally, Theorem 4 shows that the applicability of the diversification argument to the economies depicted in Figure 1 is not a coincidence. In particular, it establishes that sectoral shocks average out at the rate $\sqrt{n}$ for balanced networks in which there is a uniform bound on the degree of every sector. This result also underscores that, in contrast to some conjectures in the literature, the nature of aggregate fluctuations resulting from sectoral shocks is not related to the "sparsity" or "cyclicality" of the input-output matrix, but rather, to the extent of asymmetry between different sectors. $^{2}$

Our empirical exercise in Section 4 provides a summary of some of the relevant structural properties of the intersectoral network of the U.S. economy. We show that the empirical distributions of both first-order and second-order degrees appear to have Pareto tails, with the latter exhibiting

\footnotetext{
${ }^{2}$ The sparsity of the input-output matrix captures the fraction of its entries that are not equal to zero, while its cyclicality refers to the presence of input-output cycles in which a sector either (directly) purchases inputs from its own downstream sectors or (indirectly) from sectors that are customers of its downstream sectors (and so on).
} 
a heavier tail. Our estimates show that $\zeta=1.18$, implying that aggregate volatility in the U.S. economy decays at a rate slower than $n^{0.15}$. This substantiates our claim above that the pattern in Figure 3 is more similar to a star network than a complete network. Such a slow rate of decay - compared to the $\sqrt{n}$ convergence rate predicted by the standard diversification argument suggests that sizable aggregate fluctuations may originate from idiosyncratic shocks to different sectors in the economy.

Our paper is most closely related to Gabaix (2011), who shows that firm-level idiosyncratic shocks translate into aggregate fluctuations when the firm size distribution is sufficiently heavytailed and the largest firms contribute disproportionally to aggregate output. The intersectoral network in our model plays a role similar to that of the firm size distribution in Gabaix' analysis: shocks to sectors that take more central positions in the intersectoral network have a disproportionate effect on the aggregate output. Even though such central sectors are also larger in equilibrium, there exists important distinctions between our work and Gabaix (2011). First, in contrast to Gabaix, our focus is on the role played by input-output linkages between (disaggregated) sectors in generating aggregate fluctuations. Second, and relatedly, the sectoral (or firm) size distribution in our model is not exogenous. Rather, it is derived from these intersectoral linkages. Finally, the intersectoral network in our model also shapes the pattern of sectoral comovements. Thus, a network-based approach leads to a potentially very different behavior than an economy consisting of firms of unequal sizes, in particular, placing a range of additional restrictions on the interplay of aggregate and more micro-level data. ${ }^{3}$

Our work is also closely related to the literature on the role of sectoral shocks in macro fluctuations, such as Horvath (1998), Dupor (1999) and Shea (2002). Like these papers, we build on Long and Plosser (1983)'s multi-sectoral model of aggregate fluctuations. The debate between Horvath $(1998,2000)$ and Dupor (1999) centered around whether sectoral shocks would translate into aggregate fluctuations. Our results provide fairly complete answers to the questions raised by these papers. This literature also presents a variety of empirical evidence on the role of sectoral shocks, but does not provide a general mathematical framework similar to the one developed here. ${ }^{4}$

Our work builds on Jovanovic (1987) and Durlauf (1993), who construct models with strong strategic complementarities across firms and show that such complementarities may translate firm level shocks into volatility at the aggregate level. It is also related to Bak et al. (1993), which stresses the importance of supply chains in aggregate fluctuations. This paper provides a more comprehensive and tractable framework for the analysis of such interactions and characterizes the extent to which such interactions will impact aggregate volatility. In addition, by considering

\footnotetext{
${ }^{3}$ Foerster, Sarte, and Watson (2011) and Carvalho and Gabaix (2010) provide empirical evidence pointing to the importance of the mechanisms emphasized here. Foerster, Sarte, and Watson (2011), for example, find significant sectoral comovements consistent with the input-output structure of the economy, suggesting that the network origins of aggregate fluctuations stressed in this paper are likely to be present in practice, at least to some extent.

${ }^{4}$ Our model is also related to the smaller literature on the implications of input-output linkages on economic growth and cross-country income differences. See, for example, Ciccone (2002) and Jones (2011).
} 
richer and more complex network structures, we show how cascade effects may play a central role in translating idiosyncratic shocks into aggregate volatility.

The rest of the paper is organized as follows. Section 2 presents the basic economic environment and characterizes the influence vector, which summarizes the relevant features of the intersectoral network. Section 3 contains our main results, characterizing the relationship between the structural properties of the intersectoral network and the rate at which aggregate volatility vanishes. Section 4 illustrates the implications of our results using information from the U.S. inputoutput matrix. It also shows that second-order interconnections indeed appear to play an important role. Section 5 concludes. All proofs and some additional mathematical details are presented in the Appendix.

\section{Notation}

Throughout the paper, unless otherwise noted, all vectors are assumed to be column vectors. We denote the transpose of a matrix $X$ by $X^{\prime}$. We write $x \geq y$, if vector $x$ is element-wise greater than or equal to vector $y$. Similarly, we write $x>y$, if every element of $x$ is strictly greater than the corresponding element in $y$. We use 1 to denote the vector of all ones, the size of which is adjusted to and clear from the context. We use $\|\cdot\|_{p}$ to denote the $p$-norm of a vector as well as the induced $p$-norm of a matrix.

Given two sequences of positive real numbers $\left\{a_{n}\right\}_{n \in \mathbb{N}}$ and $\left\{b_{n}\right\}_{n \in \mathbb{N}}$, we write $a_{n}=\mathcal{O}\left(b_{n}\right)$, if they satisfy $\lim \sup _{n \rightarrow \infty} a_{n} / b_{n}<\infty$, and $a_{n}=\Omega\left(b_{n}\right)$ if $\liminf _{n \rightarrow \infty} a_{n} / b_{n}>0$. Moreover, we write $a_{n}=\Theta\left(b_{n}\right)$, if $a_{n}=\mathcal{O}\left(b_{n}\right)$ and $a_{n}=\Omega\left(b_{n}\right)$ hold simultaneously. Finally, $a_{n}=o\left(b_{n}\right)$ means that $\lim _{n \rightarrow \infty} a_{n} / b_{n}=0$.

\section{Model}

We consider a static variant of the multi-sector model of Long and Plosser (1983). The representative household is endowed with one unit of labor, supplied inelastically, and has Cobb-Douglas preferences over $n$ distinct goods; that is,

$$
u\left(c_{1}, c_{2}, \ldots, c_{n}\right)=A \prod_{i=1}^{n}\left(c_{i}\right)^{1 / n},
$$

where $c_{i}$ is the consumption of good $i$ and $A$ is a normalization constant discussed below.

Each good in the economy is produced by a competitive sector and can be either consumed by the households or used by other sectors as an input for production. The sectors use Cobb-Douglas technologies with constant returns to scale. In particular, the output of sector $i$, denoted by $x_{i}$, is

$$
x_{i}=z_{i}^{\alpha} \ell_{i}^{\alpha} \prod_{j=1}^{n} x_{i j}^{(1-\alpha) w_{i j}},
$$


where $\ell_{i}$ is the amount of labor hired by the sector, $\alpha \in(0,1)$ is the share of labor, $x_{i j}$ is the amount of commodity $j$ used in the production of good $i$, and $z_{i}$ is the idiosyncratic productivity shock to sector $i$. We assume that productivity shocks $z_{i}$ are independent across sectors, and denote the distribution of $\epsilon_{i} \equiv \log \left(z_{i}\right)$ by $F_{i}$. The exponent $w_{i j} \geq 0$ designates the share of good $j$ in the total intermediate input use of firms in sector $i . w_{i j}=0$ if sector $i$ does not use good $j$ as input for production. In view of the Cobb-Douglas technology in (2) and competitive factor markets, $w_{i j}$ also corresponds to the entries of input-output tables, measuring the value of spending on input $j$ per dollar of production of good $i$ (see Section 4). The following assumption implies that the sectoral production functions exhibit constant returns to scale: ${ }^{5}$

Assumption 1. The input shares of all sectors add up to one; that is, $\sum_{j=1}^{n} w_{i j}=1$ for all $i=$ $1,2, \ldots, n$.

We summarize the structure of intersectoral trade with the input-output matrix $W$ with enteries $w_{i j}$. Thus, the economy is completely specified by the tuple $\mathcal{E}=\left(\mathcal{I}, W,\left\{F_{i}\right\}_{i \in \mathcal{I}}\right)$, where $\mathcal{I}=\{1,2, \ldots, n\}$ denotes the set of sectors.

Input-output relationships between different sectors can be equivalently represented by a directed weighted graph on $n$ vertices, called the intersectoral network of the economy. Each vertex in this graph corresponds to a sector in the economy and a directed edge $(j, i)$ with weight $w_{i j}>0$ is present from vertex $i$ to vertex $j$ if sector $i$ is an input supplier to sector $j$. We use the notions of the intersectoral network and input-output matrix interchangeably as equivalent representations of the structure of intersectoral trades.

We also define the weighted outdegree, or simply the degree, of sector $i$ as the share of sector $i^{\prime} \mathrm{s}$ output in the input supply of the entire economy normalized by constant $1-\alpha$; that is,

$$
d_{i} \equiv \sum_{j=1}^{n} w_{j i} .
$$

Clearly, when all non-zero edge weights are identical, the outdegree of vertex $i$ is proportional to the number of sectors it is a supplier to. Finally, we refer to the collection $\left(d_{1}, d_{2}, \ldots, d_{n}\right)$ as the degree sequence of economy $\mathcal{E} .^{6}$

As we show in the appendix, in the competitive equilibrium of economy $\mathcal{E}=\left(\mathcal{I}, W,\left\{F_{i}\right\}_{i \in \mathcal{I}}\right)$, the logarithm of real value added is given by

$$
y \equiv \log (\mathrm{GDP})=v^{\prime} \epsilon,
$$

\footnotetext{
${ }^{5}$ The important but largely simplifying feature imposed by Assumption 1 is that input shares are the same across all sectors. This assumption can be relaxed without any bearing on our results, as it would be equivalent to introducing another fixed factor.

${ }^{6}$ Similarly, one can define an indegree for any given sector. However, in view of Assumption 1, the (weighted) indegrees of all sectors are equal to one. We show in Section 4 that this is a good approximation to the patterns we observe in the U.S. data.
} 
where $\epsilon \equiv\left[\epsilon_{1} \ldots \epsilon_{n}\right]^{\prime}$ and the $n$-dimensional vector $v$, called the influence vector, is defined as

$$
v \equiv \frac{\alpha}{n}\left[I-(1-\alpha) W^{\prime}\right]^{-1} \mathbf{1}
$$

Thus, the logarithm of real value added, which for simplicity we refer to as aggregate output, is a linear combination of log sectoral shocks with coefficients determined by the elements of the influence vector. Equation (4) shows that aggregate output depends on the intersectoral network of the economy through the Leontief inverse $\left[I-(1-\alpha) W^{\prime}\right]^{-1}$ (see Burress (1994)). It also captures how sectoral productivity shocks propagate downstream to other sectors through the input-output matrix. ${ }^{7}$ Finally, note that without the normalization constant $A$ in (1), the logarithm of real value added would be $y=\mu+v^{\prime} \epsilon$, where the expression for $\mu$ is provided in Appendix A. Clearly, this normalization only changes the mean of aggregate output and has no effect on its volatility or other distributional properties.

We note that the influence vector is closely related to the Bonacich centrality vector corresponding to the intersectoral network. ${ }^{8}$ Thus, sectors that take more "central" positions in the network representation of the economy play a more important role in determining aggregate output. This observation is consistent with the intuition that productivity shocks to a sector with more direct or indirect downstream customers should have more significant aggregate effects.

The vector $v$ is also the "sales vector" of the economy. In particular, as shown in Appendix A, the $i$-th element of the influence vector is equal to the equilibrium share of sales of sector $i$,

$$
v_{i}=\frac{p_{i} x_{i}}{\sum_{j=1}^{n} p_{j} x_{j}}
$$

with $p_{i}$ denoting the equilibrium price of good $i$. This is not surprising in view of the results in Hulten (1978) and Gabaix (2011), relating aggregate total factor productivity (TFP) to firm- or sector-level TFP weighted by sales. ${ }^{9}$ This observation also implies that there exists a close connection between our results on the network origins of output fluctuations and Gabaix' results on their granular origins. A major difference is that the distribution of sales shares across sectors (or other micro units) in our model is derived from input-output interactions. This not only provides microfoundations for such size differences but also enables us to sharply characterize the role of important structural properties of the network in shaping aggregate volatility. Furthermore, unlike in Gabaix (2011), the structure of interconnections also determines the comovements between

\footnotetext{
${ }^{7}$ In general, sectoral shocks also affect upstream production through a price and a quantity effect. For instance, with a negative shock to a sector, (i) its output price increases, raising its demand for inputs; and (ii) its production decreases, reducing its demand for inputs. With Cobb-Douglas production technologies, however, these two effects cancel out; see Shea (2002).

${ }^{8}$ For more on the Bonacich centrality measure, see Bonacich (1987) and Jackson (2008). For another application of this notion in economics, see Ballester, Calvó-Armengol, and Zenou (2006).

${ }^{9}$ Note that in contrast to Hulten (1978)'s formula, the logarithms of sectoral shocks (i.e, the $\epsilon$ 's) are multiplied by sales shares, and not by sales divided by value added. This is due to the fact that shocks in our model correspond to Harrod-neutral changes in productivity $\left(z_{i}=\exp \left(\epsilon_{i}\right)\right.$ is raised to the power $\left.\alpha\right)$, whereas Hulten considers Hicks-neutral changes in productivity.
} 
different sectors, placing a range of additional restrictions on the interplay of aggregate and more micro-level data (see footnote 3).

Finally, note that rather than deriving (3) and (4) as the equilibrium of a multi-sector economy, one could have started with a reduced form model $\tilde{y}=\tilde{W} \tilde{y}+\tilde{\epsilon}$, where $\tilde{y}$ is the vector consisting of the output levels, value added or other actions (or the logarithms thereof) of $n$ economic units; $\tilde{W}$ is a matrix capturing the interactions between them; and $\tilde{\epsilon}$ is a vector of independent shocks to each unit. The results presented in the remainder of the paper are applicable to any alternative model with such a representation.

\section{Network Structure and Aggregate Fluctuations}

In this section, we focus on a sequence of economies where the number of sectors increases, and characterize how the structure of the intersectoral network affects the nature of aggregate fluctuations. In particular, we consider a sequence of economies $\left\{\mathcal{E}_{n}\right\}_{n \in \mathbb{N}}$ indexed by the number of sectors $n$. The economy indexed $n$ is defined as $\mathcal{E}_{n}=\left(\mathcal{I}_{n}, W_{n},\left\{F_{i n}\right\}_{i \in \mathcal{I}_{n}}\right)$, where $\mathcal{I}_{n}=\{1,2, \ldots, n\}$ is the set of sectors in the economy; $W_{n}$ captures the corresponding input-output matrix; and $\left\{F_{i n}\right\}$ denotes the distributions of log sectoral shocks. Note that since the total supply of labor is normalized to one for all $n$, an increase in the number of sectors corresponds to disaggregating the structure of the economy. ${ }^{10}$

Given a sequence of economies $\left\{\mathcal{E}_{n}\right\}_{n \in \mathbb{N}}$, we denote the corresponding sequence of aggregate outputs and influence vectors with $\left\{y_{n}\right\}_{n \in \mathbb{N}}$ and $\left\{v_{n}\right\}_{n \in \mathbb{N}}$, respectively. We denote a generic element of the intersectoral matrix $W_{n}$ with $w_{i j}^{n}$ and the degree of sector $i$ with $d_{i}^{n}$. Finally, we denote the sequence of vectors of (log) idiosyncratic productivity shocks to the sectors with $\left\{\epsilon_{n}\right\}_{n \in \mathbb{N}}$, and impose the following assumption on their distributions:

Assumption 2. Given a sequence of economies $\left\{\mathcal{E}_{n}\right\}_{n \in \mathbb{N}}$ and for any sector $i \in \mathcal{I}_{n}, F_{\text {in }}$ is such that

(a) $\mathbb{E} \epsilon_{i n}=0$,

(b) $\operatorname{var}\left(\epsilon_{\text {in }}\right)=\sigma_{\text {in }}^{2} \in\left(\underline{\sigma}^{2}, \bar{\sigma}^{2}\right)$, where $0<\underline{\sigma}<\bar{\sigma}$ are independent of $n$.

Assumption 2(a) is a normalization. Assumption 2(b) imposes the restriction that log sectoral shock variances remain bounded as $n \rightarrow \infty$. This assumption enables us to isolate the effects of the intersectoral network structure on aggregate fluctuations as the economy gets more disaggregated, from those of the decay rate of idiosyncratic volatilities (see footnote 11).

\footnotetext{
${ }^{10}$ The fact that higher values of $n$ correspond to greater disaggregation does not put any relevant restrictions on the behavior of the sequence of input-output matrices $\left\{W_{n}\right\}_{n \in \mathbb{N}}$ for large $n$, except that the largest, second largest, thirdlargest, etc., entries of each row should be non-increasing in $n$. This does not restrict the behavior of $\left\{v_{n}\right\}_{n \in \mathbb{N}}$.
} 


\subsection{Aggregate Volatility}

Recall that aggregate output of an economy can be characterized in terms of its influence vector as $y_{n}=v_{n}^{\prime} \epsilon_{n}$. Assumption 2(a) and independence of sectoral productivity shocks imply that the standard deviation of aggregate output, which we refer to as aggregate volatility, is given by

$$
\left(\operatorname{var} y_{n}\right)^{1 / 2}=\sqrt{\sum_{i=1}^{n} \sigma_{i n}^{2} v_{i n}^{2}},
$$

where $v_{i n}$ denotes the $i$-th element of $v_{n}$. Thus, for any sequence of economies $\left\{\mathcal{E}_{n}\right\}_{n \in \mathbb{N}}$ satisfying Assumption 2(b),

$$
\left(\operatorname{var} y_{n}\right)^{1 / 2}=\Theta\left(\left\|v_{n}\right\|_{2}\right) .
$$

In other words, aggregate volatility scales with the Euclidean norm of the influence vector as our representation of the economy becomes more disaggregated. ${ }^{11}$

Though simple, this relationship shows that the rate of decay of aggregate volatility upon disaggregation may be distinct from $\sqrt{n}$ - the rate predicted by the standard diversification argument. Moreover, it also suggests that the argument for the irrelevance of sectoral shocks need not hold in general. In particular, in the extreme cases where $\left\|v_{n}\right\|_{2}$ is bounded away from zero for all values of $n$, aggregate volatility does not disappear even as $n \rightarrow \infty$. This is illustrated in the following example.

Example 1 (continued). Recall the economy depicted in Figure 2, in which sector 1 is the single input supplier of all other sectors. Using expression (4), one can verify that the corresponding influence vector is given by $v_{n}^{\prime}=\frac{\alpha}{n} \mathbf{1}^{\prime}+\left[\begin{array}{lll}(1-\alpha) & 0 \cdots 0\end{array}\right]$, implying that $\left\|v_{n}\right\|_{2}=\Theta(1)$. Thus, in view of (6), aggregate volatility does not vanish even as $n \rightarrow \infty-$ an observation consistent with the intuition discussed in the Introduction.

\subsection{Asymptotic Distributions}

Even though Example 1 shows that in the presence of strong intersectoral input-output relations, the law of large numbers may not hold, one would expect that in most realistic situations aggregate volatility vanishes as $n \rightarrow \infty .{ }^{12}$ Nevertheless, even in such sequences of economics, the network structure may have a defining effect on aggregate fluctuations. The next theorem takes a first step towards characterizing these effects by determining the asymptotic distribution of aggregate output.

\footnotetext{
${ }^{11}$ If in violation of Assumption 2(b), sectoral volatilities change at some rate $\sigma_{n}$ as $n \rightarrow \infty$, then $\left(\text { var } y_{n}\right)^{1 / 2}=$ $\Theta\left(\sigma_{n}\left\|v_{n}\right\|_{2}\right)$; that is, the rate at which aggregate volatility decays is determined by the Euclidean norm of the influence vector as well as $\sigma_{n}$. Thus, even with no intersectoral linkages, the standard diversification argument would imply that aggregate volatility decays at the rate $\sigma_{n} \sqrt{n}$.

${ }^{12}$ In particular, as Golub and Jackson (2010) show in the context of information aggregation in social networks, $\left\|v_{n}\right\|_{2} \rightarrow 0$ only if $\left\|v_{n}\right\|_{\infty} \rightarrow 0$. That is, the law of large numbers fails only if there exists some sector whose influence or sales share remains bounded away from zero even as $n \rightarrow 0$.
} 
Theorem 1. Consider a sequence of economies $\left\{\mathcal{E}_{n}\right\}_{n \in \mathbb{N}}$ and assume that $\mathbb{E} \epsilon_{i n}^{2}=\bar{\sigma}^{2}$ for all $i \in \mathcal{I}_{n}$ and all $n \in \mathbb{N}$.

(a) If $\left\{\epsilon_{i n}\right\}$ are normally distributed for all $i$ and all $n$, then $\frac{1}{\left\|v_{n}\right\|_{2}} y_{n} \stackrel{d}{\longrightarrow} \mathcal{N}\left(0, \bar{\sigma}^{2}\right)$.

(b) If $\frac{\left\|v_{n}\right\|_{\infty}}{\left\|v_{n}\right\|_{2}} \longrightarrow 0$, then $\frac{1}{\left\|v_{n}\right\|_{2}} y_{n} \stackrel{d}{\longrightarrow} \mathcal{N}\left(0, \bar{\sigma}^{2}\right)$ for all $\left\{F_{\text {in }}\right\}$.

(c) Suppose that $\left\{\epsilon_{i n}\right\}$ are identically distributed for all $i \in \mathcal{I}_{n}$ and all $n$, and are not normally distributed. If $\frac{\left\|v_{n}\right\|_{\infty}}{\left\|v_{n}\right\|_{2}} \nrightarrow 0$, then the asymptotic distribution of $\frac{1}{\left\|v_{n}\right\|_{2}} y_{n}$, when it exists, is non-normal and has finite variance $\bar{\sigma}^{2}$.

Theorem 1 establishes that aggregate output, normalized by the Euclidean norm of the influence vector, converges to a non-degenerate distribution. In other words, the rate of decay of aggregate output is determined by the same factor that captures aggregate volatility, $\left\|v_{n}\right\|_{2}$, which is itself a function of the economy's intersectoral network. It is thus a natural complement to, and strengthens, equation (6).

Theorem 1 also shows that the intersectoral structure of the economy not only affects the convergence rate, but also determines the asymptotic distribution of aggregate output: depending on $\left\|v_{n}\right\|_{\infty}$ - which captures the influence of the most influential sector - aggregate output (properly normalized) may have a non-normal distribution. In fact, as long as the conditions in part (c) of the theorem hold, the asymptotic distribution of aggregate output would necessarily depend on the specific distribution of the sectoral-level productivity shocks. In either case, however, the limiting variance of $y_{n} /\left\|v_{n}\right\|_{2}$ is finite and equal to $\bar{\sigma}^{2}$.

Finally, note that the last part of the theorem is stated conditional on the existence of such an asymptotic distribution. This assumption is necessary, as we have not assumed any restriction on the sequence of economies, and thus, $\left\|v_{n}\right\|_{\infty}$ and $\left\|v_{n}\right\|_{2}$ may not have well-behaved limits. No such assumption is required for part (b) of the theorem, which shows that any sequence of economies satisfying $\left\|v_{n}\right\|_{\infty}=o\left(\left\|v_{n}\right\|_{2}\right)$ will necessarily have a well-behaved distribution when scaled by $\left\|v_{n}\right\|_{2}$.

\subsection{First-Order Interconnections}

In the remainder of this section, we characterize the rate of decay of aggregate volatility in terms of the structural properties of the intersectoral network; properties that summarize relevant characteristics of the network without providing full details on all entries of matrix $W_{n}$.

We first focus on the effects of first-order interconnections on aggregate volatility. In particular, we show that the extent of asymmetry between sectors, measured in terms of the coefficient of variation of the degree sequence of the intersectoral network, shapes the relationship between sectoral shocks and aggregate volatility. 
Definition 1. Given an economy $\mathcal{E}_{n}$ with sectoral degrees $\left(d_{1}^{n}, d_{2}^{n}, \ldots, d_{n}^{n}\right)$, the coefficient of variation is

$$
\mathrm{CV}_{n} \equiv \frac{1}{\bar{d}_{n}}\left[\frac{1}{n-1} \sum_{i=1}^{n}\left(d_{i}^{n}-\bar{d}_{n}\right)\right]^{1 / 2}
$$

where $\bar{d}_{n}=\left(\sum_{i=1}^{n} d_{i}^{n}\right) / n$ is the average degree.

Theorem 2. Consider a sequence of economies $\left\{\mathcal{E}_{n}\right\}_{n \in \mathbb{N}}$. Then, aggregate volatility satisfies

$$
\left(\operatorname{var} y_{n}\right)^{1 / 2}=\Omega\left(\frac{1}{n} \sqrt{\sum_{i=1}^{n}\left(d_{i}^{n}\right)^{2}}\right)
$$

and

$$
\left(\operatorname{var} y_{n}\right)^{1 / 2}=\Omega\left(\frac{1+\mathrm{CV}_{n}}{\sqrt{n}}\right)
$$

Theorem 2 states that if the degree sequence of the intersectoral network exhibits high variability as measured by the coefficient of variation, then there is also high variability in the effect of different sector-specific shocks on the aggregate output. Such asymmetries in the roles of sectors imply that aggregate volatility decays at a rate slower than $\sqrt{n}$. This result also shows that the intersectoral network has a defining effect on aggregate volatility — even when the law of large numbers holds. Intuitively, when the coefficient of variation is high, only a small fraction of sectors is responsible for the majority of the input supplies in the economy. Shocks to these sectors then propagate through the entire economy as their low (resp., high) productivity leads to lower (resp., higher) production for all of their downstream sectors.

Theorem 2 also provides a more precise way of understanding the essence of the results in Example 1.

Example 1 (continued). Recall the economy with the star network representation discussed in the Introduction and depicted in Figure 2. It is easy to verify that for such an economy, $\mathrm{CV}_{n}=$ $\Theta(\sqrt{n})$. Thus, by Theorem 2, aggregate volatility is lower bounded by a constant for all values of $n$, implying that the law of large numbers fails. More generally, the theorem implies that if the economy contains a "dominant" sector whose degree grows linearly with $n$, aggregate volatility remains bounded away from zero irrespective of the level of disaggregation.

A complementary intuition for the results in Theorem 2 can be obtained from equation (7), which can also be interpreted as a condition on the tail of the empirical distribution of the degrees: aggregate volatility is higher in economies whose corresponding degree sequences have a "heavier tail". This effect can be easily quantified for intersectoral networks with power law degree sequences. 
Definition 2. A sequence of economies $\left\{\mathcal{E}_{n}\right\}_{n \in \mathbb{N}}$ has a power law degree sequence if there exist a constant $\beta>1$, a function $L(\cdot)$, and a sequence of positive numbers $c_{n}=\Theta(1)$ such that for all $n \in \mathbb{N}$ and all $k<d_{\max }^{n}=\Theta\left(n^{1 / \beta}\right)$, we have

$$
P_{n}(k)=c_{n} k^{-\beta} L(k)
$$

where $P_{n}(k) \equiv \frac{1}{n}\left|\left\{i \in \mathcal{I}_{n}: d_{i}^{n}>k\right\}\right|$ is the empirical counter-cumulative distribution function, $d_{\max }^{n}$ is the maximum degree of $\mathcal{E}_{n}$, and $L(\cdot)$ is a slowly-varying function satisfying $\lim _{t \rightarrow \infty} L(t) t^{\delta}=\infty$ and $\lim _{t \rightarrow \infty} L(t) t^{-\delta}=0$ for all $\delta>0$.

This definition is consistent with the commonly-used definition that a variable has an empirical distribution with a power law tail if $\log P(x) \simeq \gamma_{0}-\beta \log x$ for sufficiently large values of $x$. The shape parameter $\beta>1$ captures the scaling behavior of the tail of the (empirical) degree distribution: lower values of $\beta$ correspond to heavier tails and thus to larger variations in the degree sequence. Applying Theorem 2 to a sequence of economies with power law tails leads to the following corollary:

Corollary 1. Consider a sequence of economies $\left\{\mathcal{E}_{n}\right\}_{n \in \mathbb{N}}$ with a power law degree sequence and the corresponding shape parameter $\beta \in(1,2)$. Then, aggregate volatility satisfies

$$
\left(\operatorname{var} y_{n}\right)^{1 / 2}=\Omega\left(n^{-\frac{\beta-1}{\beta}-\delta}\right),
$$

where $\delta>0$ is arbitrary.

This corollary establishes that if the degree sequence of the intersectoral network exhibits relatively heavy tails, aggregate volatility decreases at a much slower rate than the one predicted by the standard diversification argument. Note that Theorem 2 and Corollary 1 provide only a lower bound on the rate at which aggregate volatility vanishes. Thus, even if the shape parameter of the power law structure is large, higher-order structural properties of the intersectoral network may still prevent the output volatility from decaying at rate $\sqrt{n}$ as we show next.

\subsection{Second-Order Interconnections and Cascades}

First-order interconnections provide only partial information about the structure of the inputoutput relationships between different sectors. In particular, as the next example demonstrates, two economies with identical degree sequences may have significantly distinct structures and thus, exhibit considerably different levels of network-originated aggregate volatility.

Example 2. Consider two sequence of economies $\left\{\mathcal{E}_{n}\right\}_{n \in \mathbb{N}}$ and $\left\{\widehat{\mathcal{E}}_{n}\right\}_{n \in \mathbb{N}}$, with corresponding intersectoral networks depicted in Figures 4(a) and 4(b), respectively. Each edge shown in the figures has weight one and all others have weight zero. Clearly, the two network structures have identical degree sequences for all $n \in \mathbb{N}$. In particular, the economy indexed $n$ in each sequence contains 
a sector of degree $d^{n}$ (labeled sector 1 ), $d^{n}-1$ sectors of degree $\tilde{d}^{n}$ (labeled 2 through $d^{n}$ ), with the rest of sectors having degrees zero. ${ }^{13}$ However, the two economies may exhibit very different levels of aggregate fluctuations.

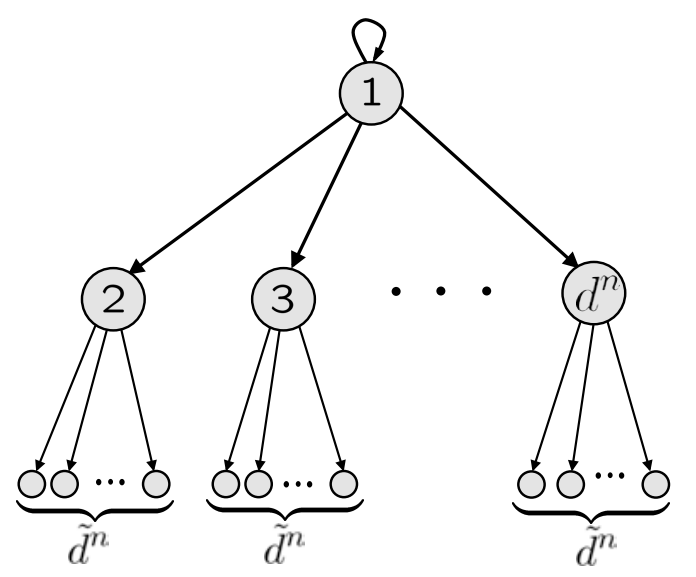

(a) $\mathcal{E}_{n}$ : high degree sectors share a common supplier

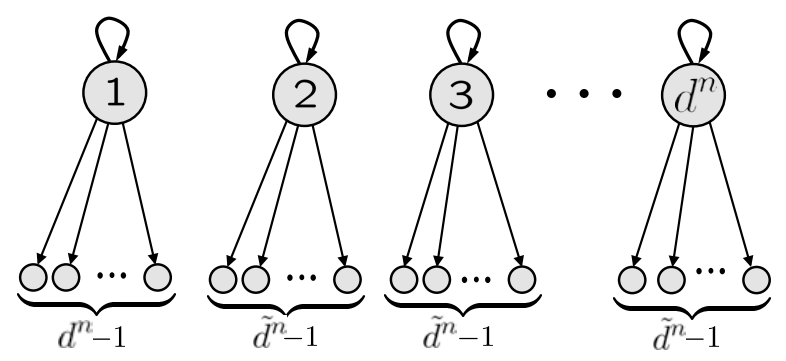

(b) $\widehat{\mathcal{E}}_{n}$ : high degree sectors do not share a common supplier

Figure 4: The two structures have identical degree sequences for all values of $n$. However, depending on the rates of $d^{n}$ and $\tilde{d}^{n}$, aggregate output volatility may exhibit considerably different behaviors for large values of $n$.

The influence vector corresponding to the sequence $\left\{\mathcal{E}_{n}\right\}_{n \in \mathbb{N}}$ depicted in Figure 4(a) is given by

$$
v_{i n}= \begin{cases}1 / n+v_{2 n}(1-\alpha)\left(d^{n}-1\right) / \alpha & \text { if } i=1 \\ \alpha / n+\alpha(1-\alpha) \tilde{d}^{n} / n & \text { if } 2 \leq i \leq d^{n} \\ \alpha / n & \text { otherwise }\end{cases}
$$

implying that $\left\|v_{n}\right\|_{2}=\Theta(1)$; i.e., aggregate volatility of $\mathcal{E}_{n}$ does not converge to zero as $n \rightarrow \infty$, regardless of the values of $d^{n}$ and $\tilde{d}^{n}$.

On the other hand, the influence vector corresponding to the sequence $\left\{\widehat{\mathcal{E}}_{n}\right\}_{n \in \mathbb{N}}$ in Figure 4 (b) is given by

$$
\widehat{v}_{i n}= \begin{cases}1 / n+(1-\alpha)\left(d^{n}-1\right) / n & \text { if } i=1 \\ 1 / n+(1-\alpha)\left(\tilde{d}^{n}-1\right) / n & \text { if } 2 \leq i \leq d^{n} \\ \alpha / n & \text { otherwise }\end{cases}
$$

implying that $\left\|\widehat{v}_{n}\right\|_{2}=\Theta\left(d^{n} / n+1 / \sqrt{d^{n}}\right)$. Thus, even though $\left\{\mathcal{E}_{n}\right\}_{n \in \mathbb{N}}$ and $\left\{\widehat{\mathcal{E}}_{n}\right\}_{n \in \mathbb{N}}$ have identical degree sequences for all $n$, the rates of decay of $\left\|v_{n}\right\|_{2}$ and $\left\|\hat{v}_{n}\right\|_{2}$ and hence, their implications for the origins of aggregate fluctuations may be very different. For example, if $d^{n}=\Theta(\sqrt{n})$, then $\left\|\widehat{v}_{n}\right\|_{2}=\Theta(1 / \sqrt[4]{n})$, whereas $\left\|v_{n}\right\|_{2}=\Theta(1)$.

\footnotetext{
${ }^{13}$ Since the total number of sectors in the economy is equal to $n$, it must be the case that $\left(d^{n}-1\right) \tilde{d}^{n}+d^{n}=n$. Such a decomposition in terms of positive integers $d^{n}$ and $\tilde{d}^{n}$ may not be possible for some $n \in \mathbb{N}$. However, the main issue discussed in this example remains valid, as only the rates of $d^{n}$ and $\tilde{d}^{n}$ as functions of $n$ matter.
} 
As Example 2 suggests, first-order interconnections in the intersectoral network provide little or no information on the extent of "cascade" effects, whereby shocks to a sector affect not only its immediate downstream sectors but also the downstream customers of those sectors and so on. Our next result provides a lower bound on the decay rate of aggregate volatility in terms of secondorder interconnections in the intersectoral network. The key concept capturing the role of such interconnections is the following new statistic.

Definition 3. Given an economy $\mathcal{E}_{n}$, the second-order interconnectivity coefficient is defined as

$$
\tau_{2}\left(W_{n}\right) \equiv \sum_{i=1}^{n} \sum_{j \neq i} \sum_{k \neq i, j} w_{j i}^{n} w_{k i}^{n} d_{j}^{n} d_{k}^{n} .
$$

This coefficient measures the extent to which sectors with high degrees (those that are major suppliers to other sectors) are interconnected to one another through common suppliers. For example, the situation with Ford, General Motors and Chrysler discussed in the Introduction corresponds to a network structure with a high second-order interconnectivity coefficient, as all three companies have high degrees (i.e., they are major suppliers and important for the economy) and rely on the same set of suppliers. More specifically, $\tau_{2}$ takes higher values when high-degree sectors share suppliers with other high-degree sectors, as opposed to low-degree ones. ${ }^{14}$ It is worth stressing that the information captured by $\tau_{2}$ is fundamentally different from the information encoded in the degree sequence of a network. We have the following result:

Theorem 3. Consider a sequence of economies $\left\{\mathcal{E}_{n}\right\}_{n \in \mathbb{N}}$. Then, aggregate volatility satisfies

$$
\left(\operatorname{var} y_{n}\right)^{1 / 2}=\Omega\left(\frac{1}{\sqrt{n}}+\frac{\mathrm{CV}_{n}}{\sqrt{n}}+\frac{\sqrt{\tau_{2}\left(W_{n}\right)}}{n}\right) .
$$

Theorem 3 shows how second-order interconnections, captured by coefficient $\tau_{2}$, affect aggregate volatility. It also shows that even if the empirical degree distributions of two sequences of economies are identical for all $n$, aggregate volatility may exhibit considerably different behavior. In this sense, Theorem 3 is a refinement of Theorem 2, taking both first and second-order relations between different sectors into account. It can also be considered to be the economically more interesting result, as it captures not only the fact that some sectors are "large" suppliers, but also the more subtle notion that there is a clustering of significant sectors, caused by the fact that they have common suppliers. Thus, in essence, Theorem 3 captures the possibility of cascade effects in the economy.

Example 2 (continued). Recall the sequences of economies $\left\{\mathcal{E}_{n}\right\}_{n \in \mathbb{N}}$ and $\left\{\widehat{\mathcal{E}}_{n}\right\}_{n \in \mathbb{N}}$ depicted in Figure 4. As mentioned earlier, intersectoral networks corresponding to the two sequences have iden-

\footnotetext{
${ }^{14}$ This observation is a consequence of the Rearrangement Inequality, which states that if $a_{1} \geq a_{2} \geq \cdots \geq a_{r}$ and $b_{1} \geq b_{2} \geq \cdots \geq b_{r}$, then for any permutation $\left(\hat{a}_{1}, \hat{a}_{2}, \ldots, \hat{a}_{r}\right)$ of $\left(a_{1}, a_{2}, \ldots, a_{r}\right)$, we have $\sum_{i=1}^{r} a_{i} b_{i} \geq \sum_{i=1}^{r} \hat{a}_{i} b_{i}$. See, e.g., Steele (2004).
} 
tical empirical degree distributions for all $n \in \mathbb{N}$. On the other hand, it is straightforward to verify that the second-order interconnectivity coefficients are very different; in particular, $\tau_{2}\left(W_{n}\right)=$ $\Theta\left(n^{2}\right)$ whereas $\tau_{2}\left(\widehat{W}_{n}\right)=0$. This is the reason behind the stark difference in the decay rate of aggregate volatility in the two sequences of economies.

Similar to the representation given in (7), we can also summarize the effects of second-order interconnection in terms of the tail of the second-order degree sequence of the economy, where the second-order degree of sector $i$ is defined as the weighted sum of the degrees of the sectors that use sector $i$ 's product as inputs with weights given by the corresponding input shares, i.e.,

$$
q_{i}^{n} \equiv \sum_{j=1}^{n} d_{j}^{n} w_{j i}^{n} .
$$

We have the following counterpart to Corollary 1.

Corollary 2. Suppose $\left\{\mathcal{E}_{n}\right\}_{n \in \mathbb{N}}$ is a sequence of economies whose second-order degree sequences have power law tails with shape parameter $\zeta \in(1,2)$ (cfr. Definition 2). Then, aggregate volatility satisfies

$$
\left(\operatorname{var} y_{n}\right)^{1 / 2}=\Omega\left(n^{-\frac{\zeta-1}{\zeta}-\delta}\right)
$$

for any $\delta>0$.

The above corollary establishes that if the distribution of second-order degrees also exhibits relatively heavy tails, then aggregate volatility decreases at a much slower rate than the one predicted by the standard diversification argument. As Example 2 shows, second-order effects may dominate the first-order effect of the degree distribution in determining the decay rate of aggregate volatility of the economy. In particular, for a sequence of economies in which the empirical distributions of both first- and second-order degrees exhibit power law tails with exponents $\beta$ and $\zeta$, the tighter bound for the decay rate of aggregate volatility is determined by $\min \{\beta, \zeta\}$.

The results in Theorem 3 can be further strengthened to capture the effects of higher-order interconnections and more complex patterns of cascades. We provide such a characterization in Appendix D, corresponding to tighter lower bounds on the decay rate of aggregate volatility.

\subsection{Balanced Structures}

Finally, we establish a partial converse to Theorem 2, showing that with limited variations in the degrees of different sectors, aggregate volatility decays at rate $\sqrt{n}$ - consistent with the standard diversification argument.

Definition 4. A sequence of economies $\left\{\mathcal{E}_{n}\right\}_{n \in \mathbb{N}}$ is balanced if $\max _{i \in \mathcal{I}_{n}} d_{i}^{n}=\Theta(1)$. 
In balanced structures, there is a limit on the extent of asymmetry in the importance of different sectors as suppliers, in the sense that no sector experiences an unbounded increase in its degree as $n \rightarrow \infty$. Thus, balanced structures can be considered as the polar opposites of network structures with "dominant" sectors - sectors whose degrees grow linearly as $n \rightarrow \infty$ - such as the one in Figure 2 .

Theorem 4. Consider a sequence of balanced economies $\left\{\mathcal{E}_{n}\right\}_{n \in \mathbb{N}}$. Then there exists $\bar{\alpha} \in(0,1)$ such that for $\alpha \geq \bar{\alpha},\left(\operatorname{var} y_{n}\right)^{1 / 2}=\Theta(1 / \sqrt{n})$.

This theorem shows that when the intersectoral network has a balanced structure and the role of the intermediate inputs in production is not too large, volatility decays at rate $\sqrt{n}$, implying that other structural properties of the network cannot contribute any further to aggregate volatility. Consequently, in economies with balanced intersectoral network structures, aggregate fluctuations do not have network origins.

A noteworthy corollary to this theorem is that many network structures that are often considered to be "fragile", such as the ring and the binary tree depcited in Figure 5, have exactly the same asymptotic behavior as the structures in Figure 1 as far as aggregate volatility is concerned. In fact, the "sparseness" and "cyclicality" of the input-output matrix has no impact on this asymptotic behavior. It is only in network structures with asymmetric roles for different sectors - either in terms of first-order or higher-order interconnections - that sectoral (or more micro) shocks can be the origins of aggregate fluctuations.

Theorem 4 is a generalization of the results of Dupor (1999). As noted by Dupor and Horvath (1998) in a related context, Theorem 4 is both an aggregation and an irrelevance result for

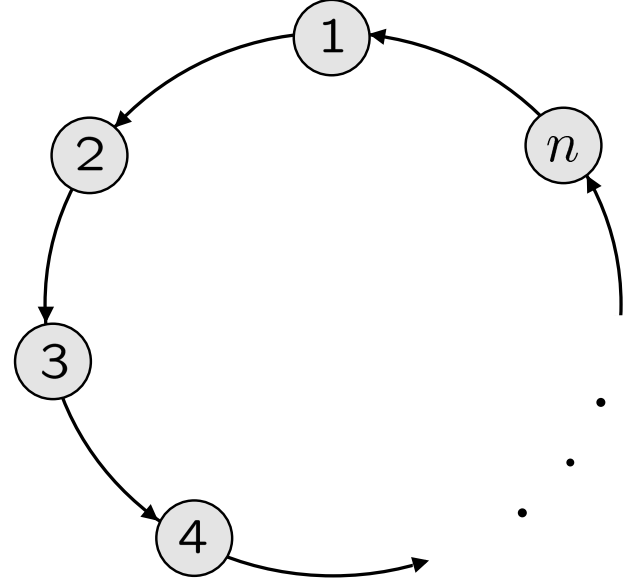

(a) The ring

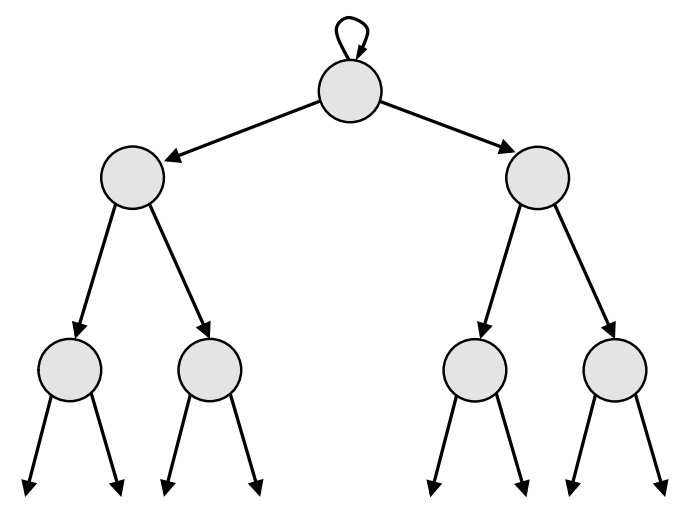

(b) The binary tree

Figure 5: Economies with balanced intersectoral network structures: aggregate volatility decays at rate $\sqrt{n}$. 
economies with balanced structures. As an aggregation result it suggests an observational equivalence between the single aggregate sector economy and the multi-sector economy where the variance of sector-specific shocks scales by the level of disaggregation. On the other hand, as an irrelevance result, it shows that within the class of balanced structures, different input-output matrices generate roughly the same amount of volatility. However, note that in contrast to the claims in Lucas (1977) and Dupor (1999), our earlier results clearly establish that neither the aggregation nor the irrelevance interpretations hold for more general intersectoral networks.

\section{Application}

In this section, we briefly look at the intersectoral network structure of the U.S. economy and study its implications for aggregate fluctuations as predicted by our results in Section 3. For this purpose, we use the detailed benchmark input-output accounts spanning the 1972-2002 period, compiled every five years by the Bureau of Economic Analysis. We use commodity-by-commodity direct requirements tables where the typical $(i, j)$ entry gives the input-share (evaluated at current producers' prices) of commodity $i$ as a fraction of total output of commodity $j .{ }^{15}$ These detailed input-output accounts constitute the finest level of disaggregation available for the U.S. intersectoral trade data, with most sectors (roughly) corresponding to four-digit S.I.C. definitions. ${ }^{16}$ Even though we consider our results applicable at a finer level than that available through the BEA tables, this exercise is useful to obtain a rough empirical grounding for our results. Moreover, it enables us to perform back-of-the-envelope calculations to get an impression of the role played by the U.S. input-output structure in the relationship between sectoral shocks and aggregate volatility.

We start by analyzing the variation in total intermediate input shares across commodities, or equivalently, the variation in the (weighted) indegrees for each sector. The first panel of Figure 6 shows the non-parametric estimate of the empirical density of intermediate input shares for 2002. The second panel displays the same densities for every detailed direct requirements table since 1972. ${ }^{17}$ In line with the previous estimates of Basu (1995) and Jones (forthcoming), by averaging across years and sectors we find an intermediate input share of 0.55 . This average share is stable over time, ranging between a minimum of 0.52 in 1987 and a maximum of 0.58 in 2002. There exists some variation across sectors, as some are more (intermediate) input-intensive than others. Yet, the indegree of most sectors concentrate around the mean: on average 71 percent of the sectors

\footnotetext{
${ }^{15}$ By slightly abusing terminology, we use the terms commodity and sector interchangeably throughout this section.

${ }^{16}$ The BEA tables for the period 1972-1992 are based on an evolving S.I.C. classification, whereas the NAICS system was adopted from 1997 onwards. While individual sectors are not immediately comparable across S.I.C. and NAICS classifications, the corresponding intersectoral network structures - the objects of analysis in this paper - will be shown to be remarkably stable.

${ }^{17}$ We used the Gaussian distribution as the kernel smoothing function with a bandwidth of 0.3 .
} 

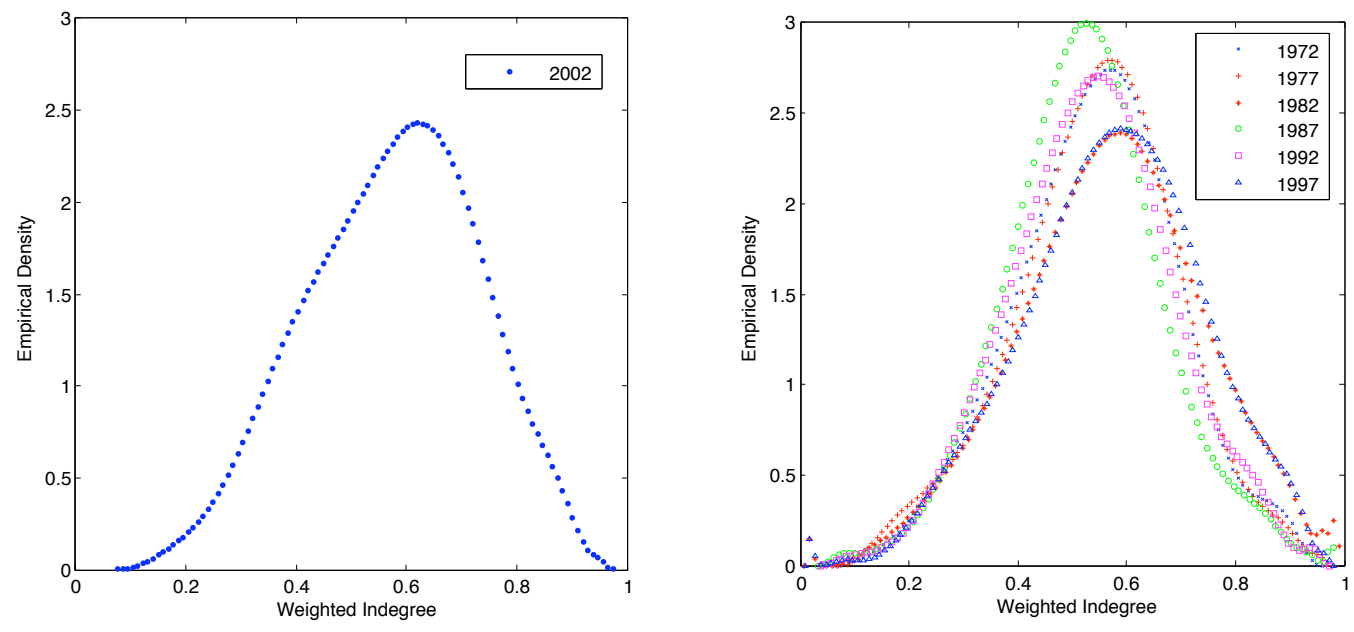

Figure 6: Empirical densities of intermediate input shares (indegrees)

are within one standard deviation of the mean input share. ${ }^{18}$

Recall that in our model we assumed that the intermediate input share is the same and equal to $1-\alpha$ across all sectors. Thus, in order to obtain the data counterpart of our $W$ matrix, we renormalized each entry in the direct requirements tables by the total input requirement of the corresponding sector and then computed the corresponding first and second-order degrees, $d_{i}$ and $q_{i}$ respectively. ${ }^{19}$ Figure 7 shows the non-parametric estimates of the corresponding empirical densities in $2002 .^{20}$

Unlike their indegree counterpart, the empirical distributions of both first and second-order
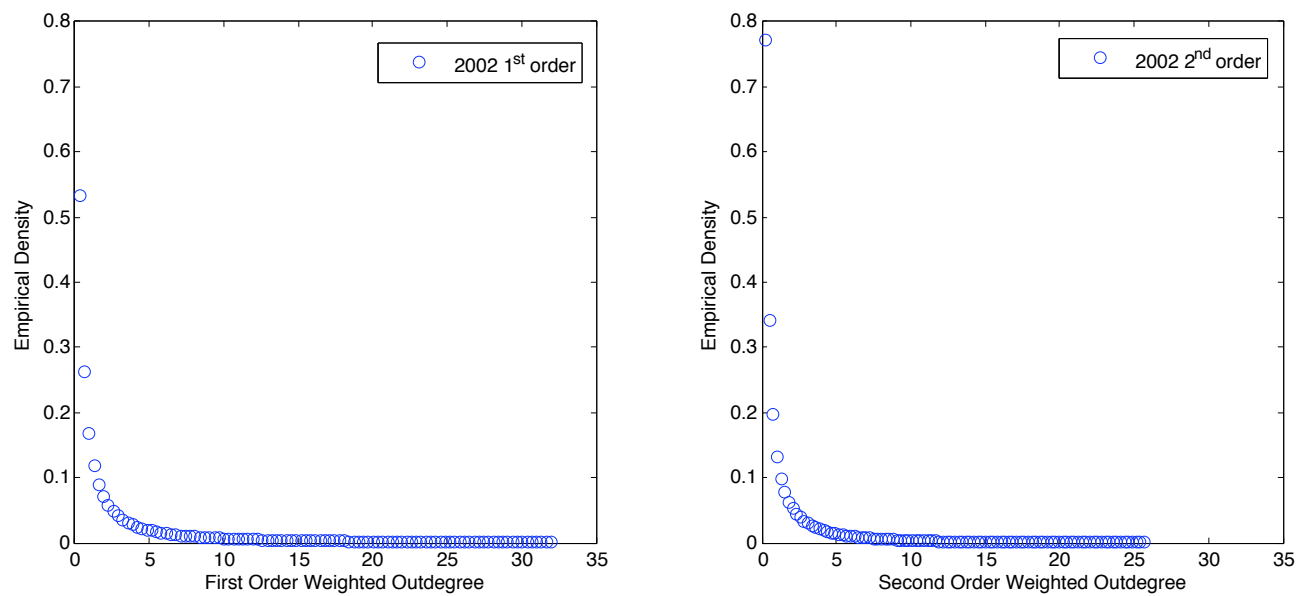

Figure 7: Empirical densities of first and second-order degrees

\footnotetext{
${ }^{18}$ This is again stable over different years, ranging from 0.74 in 1977 to 0.67 in 2002 . The equivalent two standard deviation number is 0.95 .

${ }^{19}$ We checked that all results below still apply when we do not perform this normalization.

${ }^{20}$ In Figure 7, we excluded commodities with zero outdegree, i.e., those that do not enter as intermediate inputs in the production of other commodities.
} 
(out)degrees are noticeably skewed, with heavy right tails. Such skewed distributions are, respectively, indicative of presence of commodities that are (i) general purpose inputs used by many other sectors; (ii) major suppliers to sectors that produce the general purpose inputs. ${ }^{21}$ In either case, the fraction of commodities whose weighted first-order (resp., second-order) degrees are an order of magnitude above the mean first-order (resp., second-order) degree is non-negligible. ${ }^{22}$

To further characterize such heavy tailed behaviors, Figures 8 and 9 plot the empirical countercumulative distribution functions (i.e., one minus the empirical cumulative distribution functions) of the first-order and second-order degrees on a log-log scale. The first panels in both figures also show non-parametric estimates for the empirical counter-cumulative distributions in 2002 using the Nadaraya-Watson kernel regression with a bandwidth selected using least squares crossvalidation (Nadaraya (1964) and Watson (1964)). The second panels show the empirical countercumulative distributions for all other years. In either case, the tail of the distribution is wellapproximated by a power law distribution as shown by the approximate linear relationship.

An estimate for the shape parameters can in principle be obtained by running an ordinary least squares (OLS) regression of the empirical log-CCDF on the log-outdegree sequence. However, as Gabaix and Ibragimov (2011) point out, these simple OLS estimates are downward biased in small samples. Thus, to account for this bias, we implement the modified log rank-log size regression suggested by Gabaix and Ibragimov. Throughout, we take the tail of the counter-cumulative distributions to correspond to the $20 \%$ largest sectors in terms of $d$ and $q$. The resulting estimates
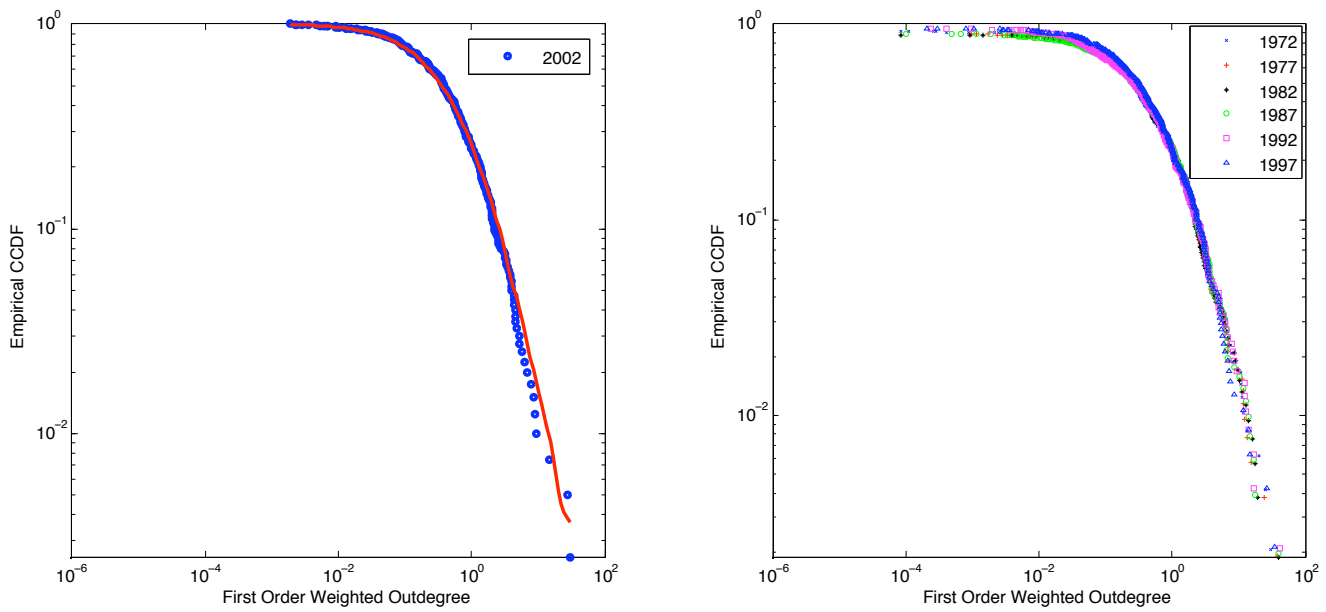

Figure 8: Empirical counter-cumulative distribution function of first-order degrees

\footnotetext{
${ }^{21}$ The top five sectors with the highest first-order degrees are: management of companies and enterprises, wholesale trade, real estate, electric power generation, transmission, and distribution, and iron and steel mills and ferroalloy manufacturing. The top five sectors with the highest second-order degrees are: management of companies and enterprises, wholesale trade, real estate, advertising and related services, and monetary authorities and depository credit intermediation.

${ }^{22}$ Note that, given the normalization discussed above, the mean first-order and second-order degrees are necessarily equal to one.
} 

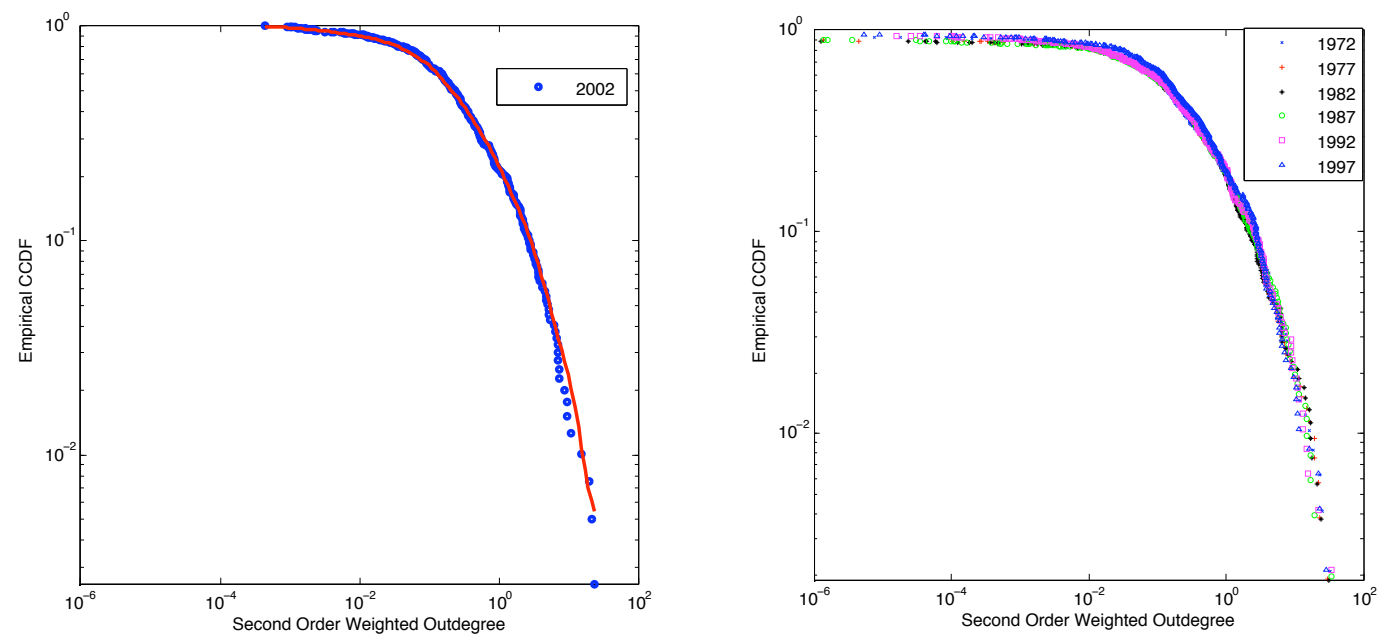

Figure 9: Empirical counter-cumulative distribution function of second-order degrees

are shown in Table 1 along with the corresponding standard errors. Notice that the estimates for the shape parameter of the first order-degree distribution are always above the corresponding estimates for the second-order degree distribution. Averaging across years, the OLS estimates give $\widehat{\beta}=1.38$ for the first-order degree and $\widehat{\zeta}=1.18$ for the second-order degree.

\begin{tabular}{cccccccc}
\hline \hline & 1972 & 1977 & 1982 & 1987 & 1992 & 1997 & 2002 \\
\hline$\widehat{\beta}$ & 1.38 & 1.38 & 1.35 & 1.37 & 1.32 & 1.43 & 1.46 \\
& $(0.20 ; 97)$ & $(0.19 ; 105)$ & $(0.18 ; 106)$ & $(0.19 ; 102)$ & $(0.19 ; 95)$ & $(0.21 ; 95)$ & $(0.23 ; 83)$ \\
$\widehat{\zeta}$ & 1.14 & 1.15 & 1.10 & 1.14 & 1.15 & 1.27 & 1.30 \\
& $(0.16 ; 97)$ & $(0.16 ; 105)$ & $(0.15 ; 106)$ & $(0.16 ; 102)$ & $(0.17 ; 95)$ & $(0.18 ; 95)$ & $(0.20 ; 83)$ \\
$n$ & 483 & 524 & 529 & 510 & 476 & 474 & 417 \\
\hline
\end{tabular}

Table 1: OLS estimates of $\beta$ and $\zeta$ using Gabaix and Ibragimov (2011) correction. The numbers in parenthesis denote the associated standard errors and the number of observations corresponding to the $20 \%$ largest sectors, which are the ones used in the estimation of the shape parameter. The last row shows the total number of sectors for that year.

As a cross-check, we also calculated the average slope implied by the non-parametric NadarayaWatson regression, while again taking the tail to correspond to $20 \%$ of the samples in each year. Averaging over years, the absolute values of the implied slopes are 1.28 and 1.17 for the first and second-order degree distributions respectively, which are fairly close to the OLS estimates. As yet another alternative, we also calculated Hill-type MLE estimates of $\beta$ and $\zeta$. In particular, we followed Clauset, Shalizi, and Newman (2009) in using all observations on or above some endogenously determined cut-off point. Averaging across years, these MLE estimates are $\widehat{\beta}=1.39$ and $\widehat{\zeta}=1.14$ which are again very close to the baseline OLS estimates reported above. ${ }^{23}$

\footnotetext{
${ }^{23}$ The impact of normalizing the input shares is also negligible for these estimates. Using the original directrequirements tables to compute first and second order degrees gives average OLS estimates of $\widehat{\beta}=1.42$ and $\widehat{\zeta}=1.23$.
} 
These estimates imply that there exists a high degree of asymmetry in the U.S. economy in terms of the roles that different sectors play as direct or indirect suppliers to others; suggesting the potential presence of network-originated aggregate fluctuations. Computing $\left\|v_{n}\right\|_{2}$ for the U.S. input-output matrix gives a value twice as large as $\sqrt{n}$, which suggests that, at this level of disaggregation, intersectoral linkages increase the impact of sectoral shocks by at least twofold. However, since the sectors in the U.S. input-output matrix are highly aggregated, this comparison provides only limited information on the importance of microeconomic shocks (to disaggregated sectors) in generating aggregate fluctuations. We can get a (preliminary) sense of the impact of microeconomic shocks on aggregate volatility at finer levels of disaggregations by extrapolating from the distribution of first-order and second-order degrees in Figures 8 and 9. Clearly, we only observe the input-output matrix - the equivalent of matrix $W$ in our model - for a single economy, rather than a sequence of economies. Therefore, such extrapolations are inevitably speculative. Nevertheless, the "scale-free" nature of the power law distribution, which appears to be a good approximation to the data, suggests that the tail behavior of first-order and second-order degrees may be informative about their behavior at higher levels of disaggregation, which is the input we need for this quantitative exercise.

As suggested by the discussion following Corollary 2, the estimates in Table 1 imply that the lower bound on the rate of decay of standard deviation obtained from the second-order degrees is considerably tighter than that obtained from the first-order degrees. In particular, the shape parameter $\widehat{\zeta}=1.18$ implies that aggregate volatility decays no faster than $n^{(\zeta-1) / \zeta}=n^{0.15}$, whereas the lower bound implied by the average shape parameter for the first-order degrees, $\widehat{\beta}=1.38$, is $n^{(\beta-1) / \beta}=n^{0.28}$. It is worth noting that this is a significantly slower rate of decay than $\sqrt{n}-$ the rate predicted by the standard diversification argument.

To gain a further understanding of the implications of this rate of decay, we computed the (over time) average standard deviation of total factor productivity across 459 four-digit (SIC) manufacturing industries from the NBER productivity database between 1958 and 2005 (after controlling for a linear time trend to account for the secular decline in several manufacturing industries). ${ }^{24}$ This average standard deviation is estimated as $0.058 .{ }^{25}$ On the other hand, over the same time period, the average of the U.S. GDP accounted for by manufacturing is around $20 \% .{ }^{26}$ Thus if, for the purpose of our back-of-the-envelope calculations, we assume that the 459 four-digit manufacturing sectors correspond to 1/5th of the GDP, we can consider that the economy comprises $5 \times 459=2295$ sectors at the same level of disaggregation as four-digit manufacturing indus-

\footnotetext{
${ }^{24}$ To the extent that total factor productivity is measured correctly, it approximates the variability of idiosyncratic sectoral shocks. In contrast, the variability of sectoral value added is determined by idiosyncratic shocks as well as the sectoral linkages, as we emphasized throughout the paper.

${ }^{25}$ If we instead weigh different industries by the logarithm of their value added so that small industries do not receive disproportionate weights, the average becomes 0.054 and does not change by much.

${ }^{26}$ Data from http://www.bea.gov/industry/gdpbyind_data.htm.
} 
tries. ${ }^{27}$ With a sectoral volatility of 0.06 , if aggregate volatility decayed at the rate $\sqrt{n}$, as would have been the case with a balanced structure, we would expect it to be approximately around $0.058 / \sqrt{2295} \simeq 0.001$; clearly corresponding to a very small amount of variability. This observation underscores the fact that in a balanced structure with a reasonably large number of sectors, sector-specific shocks average out and do not translate into a sizable amount of aggregate volatility. If, instead, as suggested by our lower bound from the second-order degree distribution, aggregate volatility decays at the rate $n^{0.15}$, the same number would be $0.058 /(2295)^{0.15} \simeq 0.018$. This corresponds to sizable aggregate fluctuations, in the ballpark of the approximately $2 \%$ standard deviation of the U.S. GDP.

These back-of-the-envelope calculations based on the second approach thus suggest that the types of interconnections implied by the U.S. input-output structure may generate significant aggregate fluctuations from sectoral shocks. Note that - as we have already emphasized - these calculations are merely suggestive and are no substitute for a systematic econometric and quantitative investigation of the implications of the input-output linkages in the U.S. economy, which we leave for future work.

\section{Conclusion}

The general consensus in macroeconomics has been that microeconomic shocks to firms or disaggregated sectors cannot generate significant aggregate fluctuations. This consensus, based on a "diversification argument," has maintained that such "idiosyncratic" shocks would wash out as aggregate output concentrates around its mean at the very rapid rate of $\sqrt{n}$.

This paper argues that in the presence of intersectoral input-output linkages, such a diversification argument may not apply. Rather, propagation of microeconomic idiosyncratic shocks due to the intersectoral linkages may indeed lead to aggregate fluctuations. Moreover, the paper shows that the rate at which aggregate volatility decays explicitly depends on the structure of the intersectoral network representing input-output linkages. Our results provide a characterization of this relationship in terms of the importance of different sectors as direct or indirect suppliers to the rest of the economy. In particular, we show that high levels of variability in the degrees of different sectors (as captured by the corresponding coefficient of variation) as well as the presence of high degree sectors that share common suppliers (as measured by the second-order interconnectivity coefficient) imply slower rates of decay for aggregate volatility.

The main insight suggested by this paper is that sizable aggregate fluctuations may originate from microeconomic shocks only if there are significant asymmetries in the roles that sectors play

\footnotetext{
${ }^{27}$ One might be concerned that manufacturing is more volatile than non-manufacturing. This does not appear to be the case, however, at the three-digit level, where we can compare manufacturing and non-manufacturing industries. If anything, manufacturing industries appear to be somewhat less volatile with or without controlling for industry size (though this difference is not statistically significant in either case).
} 
as direct or indirect suppliers to others. This analysis provides a fairly complete answer to the debate between Dupor (1999) and Horvath (1998, 2000). It shows that while Dupor's critique applies to economies with balanced structures, in general the sectoral structure of the economy may have a defining impact on aggregate fluctuations.

Our analysis suggests a number of directions for future research. First, a more systematic analysis to investigate the quantitative importance of the mechanisms stressed in this paper is required. In this vein, Carvalho (2008) extends our characterization to the class of dynamic multisector economies considered by Long and Plosser (1983) and Horvath (2000), and conducts a detailed calibration exercise to show that the intersectoral network structure of the U.S. economy can account for a large fraction of observed sectoral comovement and aggregate volatility of the U.S. economy. Such an investigation can be complemented by systematic econometric analyses that build on Foerster, Sarte, and Watson (2011) and exploit both the time-series and cross-sectoral implications of the approach developed here.

Second, the characterization results provided here focus on the standard deviation of log value added, which captures the nature of fluctuations "near the mean" of aggregate output. This can be supplemented by a systematic analysis of large deviations of output from its mean. Acemoglu, Ozdaglar, and Tahbaz-Salehi (2010) provide a first set of results relating the likelihood of tail events to the structural properties of the intersectoral network.

Third, throughout the paper we assumed that the intersectoral network captures the technological constraints of different sectors and is exogenously given. In practice, however, input-output linkages between different firms and disaggregated sectors are endogenously determined. For example, by making costly investments in building relationships with several suppliers, firms may be able to reduce their inputs' volatilities, creating a trade-off. Part of this trade-off will be shaped by how risky different suppliers are perceived to be and how risk is evaluated and priced in the economy. Characterizing the implications of such trade-offs is an important direction for future research.

Last but not least, another important area for future research is a systematic analysis of how shocks are transmitted within financial networks, clarifying which types of financial network structures are more susceptible to negative shocks to specific financial institutions. 


\section{Appendix A: Competitive Equilibrium}

Definition. A competitive equilibrium of economy $\mathcal{E}$ with $n$ sectors consists of prices $\left(p_{1}, p_{2}, \ldots, p_{n}\right)$, wage $h$, consumption bundle $\left(c_{1}, c_{2}, \ldots, c_{n}\right)$, and quantities $\left(\ell_{i}, x_{i},\left(x_{i j}\right)\right)$ such that

(a) the representative consumer maximizes her utility,

(b) the representative firms in each sector maximize profits,

(c) labor and commodity markets clear, i.e.,

$$
\begin{aligned}
c_{i}+\sum_{j=1}^{n} x_{j i} & =x_{i} \quad \forall i=1, \ldots, n \\
\sum_{i=1}^{n} \ell_{i} & =1 .
\end{aligned}
$$

Taking first-order conditions with respect to $\ell_{i}$ and $x_{i j}$ in firm $i$ 's problem and substituting the resulting demands in its production technology yields

$$
\alpha \log (h)=\alpha \epsilon_{i}+B+\log \left(p_{i}\right)-(1-\alpha) \sum_{j=1}^{n} w_{i j} \log \left(p_{j}\right)+(1-\alpha) \sum_{j=1}^{n} w_{i j} \log \left(w_{i j}\right)
$$

where $B$ is a constant given by $B=\alpha \log (\alpha)+(1-\alpha) \log (1-\alpha)$. Multiplying the above equation by the $i$-th element of the influence vector $v^{\prime}=\frac{\alpha}{n} \mathbf{1}^{\prime}[I-(1-\alpha) W]^{-1}$ and summing over all sectors $i$ lead to

$$
\log (h)=v^{\prime} \epsilon+\mu
$$

where $\mu$ is a constant independent of the vector of shocks $\epsilon$ and is given by

$$
\mu=\frac{1}{n} \sum_{i=1}^{n} \log \left(p_{i}\right)+B / \alpha+\frac{1-\alpha}{\alpha} \sum_{i=1}^{n} \sum_{j=1}^{n} v_{i} w_{i j} \log \left(w_{i j}\right) .
$$

Finally, by setting

$$
A=n \exp \left(-B / \alpha-\frac{(1-\alpha)}{\alpha} \sum_{i=1}^{n} \sum_{j=1}^{n} v_{i} w_{i j} \log \left(w_{i j}\right)\right)
$$

and normalizing the ideal price index to 1 , i.e., $\frac{n}{A}\left(p_{1} p_{2} \ldots p_{n}\right)^{1 / n}=1$, we obtain

$$
y=\log (h)=v^{\prime} \epsilon
$$

That is, the logarithm of real value added in a given economy, aggregate output, is simply a weighted sum of sector-specific productivity shocks, where the weights are determined by the corresponding influence vector. 
We now show that the influence vector also captures the equilibrium share of sales of different sectors. By plugging in labor and input demands, and consumption levels into the market clearing condition for commodity $i$, we obtain $h / n+(1-\alpha) \sum_{j=1}^{n} w_{j i} p_{j} x_{j}=p_{i} x_{i}$. This implies that $s_{i}=h / n+(1-\alpha) \sum_{j=1}^{n} s_{j} w_{j i}$, where $s_{i}=p_{i} x_{i}$ is the equilibrium value of sales of sector $i$. Thus, the vector of equilibrium sales is related to the influence vector through $s^{\prime}=$ $(h / n) \mathbf{1}^{\prime}[I-(1-\alpha) W]^{-1}=(h / \alpha) v^{\prime}$. Therefore,

$$
v_{i}=\frac{p_{i} x_{i}}{\sum_{j=1}^{n} p_{j} x_{j}}
$$

where we have used the fact that $v_{n}^{\prime} 1=1$.

The relationship between equilibirum shares of sales of different sectors and the influence vector can also be derived directly by applying a variant of Hulten (1978)'s theorem, which establishes that if the production functions are given by $x_{i}=e^{\alpha \epsilon_{i}} f\left(x_{i 1}, \ldots, x_{i n}, \ell_{i}\right)$, a productivity change of $d\left(\alpha \epsilon_{i}\right)$ to sector $i$ causes an increase in GDP equal to

$$
d(\mathrm{GDP})=\frac{p_{i} x_{i}}{\mathrm{GDP}} d\left(\alpha \epsilon_{i}\right) .
$$

Finally, the fact that $h=\alpha \sum_{i=1}^{n} p_{i} x_{i}$ implies

$$
v_{i}=\frac{d h}{d \epsilon_{i}}=\frac{p_{i} x_{i}}{\sum_{j=1}^{n} p_{j} x_{j}} .
$$

\section{Appendix B: Central Limit Theorems}

\section{The Lindeberg-Feller Theorem}

The Lindeberg-Feller Theorem (Durrett (2005, p. 114)) provides sufficient conditions under which the distribution of sums of independent, but not necessarily identically distributed random variables converges to the normal law.

Theorem A. 1 (Lindeberg-Feller). Consider the triangular array of independent random variables $\xi_{\text {in, }}$ $1 \leq i \leq n$, with zero expectations and finite variances such that

$$
\sum_{i=1}^{n} \mathbb{E} \xi_{i n}^{2}=1 .
$$

Also suppose that Lindeberg's condition holds, i.e.,

$$
\lim _{n \rightarrow \infty} \sum_{i=1}^{n} \mathbb{E}\left(\xi_{i n}^{2} \mathbb{I}_{\left\{\left|\xi_{i n}\right|>\delta\right\}}\right)=0 \quad \text { for all } \delta>0,
$$

where II denotes the indicator function. Then,

$$
\xi_{1 n}+\xi_{2 n}+\cdots+\xi_{n n} \stackrel{d}{\longrightarrow} \mathcal{N}(0,1) .
$$




\section{Non-Classical Central Limit Theorems}

To establish asymptotic normality for triangular arrays of random variables $\left\{\xi_{\text {in }}\right\}$ that do not satisfy Lindeberg's condition (13), one needs to apply "non-classical" generalizations of the central limit theorem. The following theorem is from Rotar (1975). A detailed treatment of the subject can be found in Chapter 9 of Linnik and Ostrovskir (1977).

Theorem A.2. Consider a triangular array of independent random variables $\xi_{i n}, 1 \leq i \leq n$, with distributions $G_{i n}$, zero expectations, and finite variances $\sigma_{i n^{\prime}}^{2}$ such that $\sum_{i=1}^{n} \sigma_{i n}^{2}=1$. Then $\sum_{i=1}^{n} \xi_{\text {in }} \longrightarrow \mathcal{N}(0,1)$ in distribution, only if

$$
\lim _{n \rightarrow \infty} \sum_{i=1}^{n} \int_{|t|>\delta}|t|\left|G_{i n}(t)-\Phi_{i n}(t)\right| d t=0 \quad \text { for all } \delta>0,
$$

where $\Phi_{i n}(t)=\Phi\left(t / \sigma_{i n}\right)$ and $\Phi$ denotes the standard normal distribution.

\section{Appendix C: Proofs}

Throughout the proofs, for notational simplicity, we drop the index $n$ when denoting the degrees of different sectors and the elements of matrix $W_{n}$ if no confusion arises.

Proof of Theorem 1: The proof of part (a) is trivial and is omitted.

In order to prove part (b), define the triangular array of real numbers $\left\{\xi_{i n}\right\}_{1 \leq i \leq n}$ as $\xi_{\text {in }}=$ $v_{i n} \epsilon_{i n} / \bar{\sigma}\left\|v_{n}\right\|_{2}$. By definition, $\frac{1}{\bar{\sigma}\left\|v_{n}\right\|_{2}} y_{n}=\xi_{1 n}+\cdots+\xi_{n n}$. It is straightforward to verify that the following relations hold:

$$
\begin{aligned}
\mathbb{E} \xi_{\text {in }} & =0 . \\
\sum_{i=1}^{n} \mathbb{E} \xi_{\text {in }}^{2} & =1 .
\end{aligned}
$$

Therefore, by the Lindeberg-Feller Theorem, $y_{n} / \bar{\sigma}\left\|v_{n}\right\|_{2}$ converges in distribution to the standard normal law, provided that Lindeberg's condition (13) is satisfied. In order to verify that Lindeberg's condition indeed holds, notice that we have,

$$
\begin{aligned}
\sum_{i=1}^{n} \mathbb{E}\left(\xi_{i n}^{2} \mathbb{I}_{\left\{\left|\xi_{i n}\right|>\delta\right\}}\right) & =\frac{1}{\bar{\sigma}^{2}\left\|v_{n}\right\|_{2}^{2}} \sum_{i=1}^{n} v_{i n}^{2} \mathbb{E}\left[\epsilon_{i n}^{2} \mathbb{I}_{\left.\left\{\left|\epsilon_{i n}\right|>\frac{\delta \overline{\|}\left\|v_{n}\right\|_{2}}{\left|v_{i n}\right|}\right\}\right]}\right] \\
& \leq \frac{1}{\bar{\sigma}^{2}\left\|v_{n}\right\|_{2}^{2}} \sum_{i=1}^{n} v_{i n}^{2} \mathbb{E}\left[\epsilon_{i n}^{2} \mathbb{I}_{\left.\left\{\left|\epsilon_{i n}\right|>\frac{\delta \overline{\|}\left\|v_{n}\right\|_{2}}{\left\|v_{n}\right\|_{\infty}}\right\}\right]}\right] \\
& =\frac{1}{\bar{\sigma}^{2}} \mathbb{E}\left[\epsilon_{i n}^{2} \mathbb{I}_{\left.\left\{\left|\epsilon_{i n}\right|>\frac{\delta \bar{\delta}\left\|v_{n}\right\|_{2}}{\left\|v_{n}\right\|_{\infty}}\right\}\right] .}\right.
\end{aligned}
$$


By the dominated convergence theorem and the assumption that $\left\|v_{n}\right\|_{\infty}=o\left(\left\|v_{n}\right\|_{2}\right)$, the right-hand side of the above equality converges to zero as $n \rightarrow \infty$, and therefore,

$$
\lim _{n \rightarrow \infty} \sum_{i=1}^{n} \mathbb{E}\left(\xi_{i n}^{2} \mathbb{I}_{\left\{\left|\xi_{i n}\right|>\delta\right\}}\right)=0 \quad \text { for all } \delta>0
$$

completing the proof of part (b).

Finally, in order to prove part (c), we show that the triangular array of random variables $\xi_{\text {in }}=$ $\epsilon_{i n} v_{i n} / \bar{\sigma}\left\|v_{n}\right\|_{2}$ does not satisfy condition (14). ${ }^{28}$ The distribution function of $\xi_{\text {in }}$ is given by $G_{i n}(t)=$ $F\left(t \bar{\sigma}\left\|v_{n}\right\|_{2} / v_{i n}\right)$, where $F$ denotes the distribution of $\epsilon_{i n}$. Therefore,

$$
\begin{aligned}
\sum_{i=1}^{n} \int_{|t|>\delta}|t|\left|G_{i n}(t)-\Phi_{i n}(t)\right| d t & =\frac{1}{\bar{\sigma}^{2}\left\|v_{n}\right\|_{2}^{2}} \sum_{i=1}^{n} v_{i n}^{2} \int_{-\infty}^{\infty}|s||F(s)-\Phi(s)| \mathbb{I}_{\left\{|s|>\frac{\delta \bar{\sigma}\left\|v_{n}\right\|_{2}}{\left|v_{i n}\right|}\right\}} d s \\
& \geq\left(\frac{\left\|v_{n}\right\|_{\infty}}{\bar{\sigma}\left\|v_{n}\right\|_{2}}\right)^{2} \int_{-\infty}^{\infty}|s \| F(s)-\Phi(s)| \mathbb{I}_{\left\{|s|>\delta \bar{\sigma}\left\|v_{n}\right\|_{2}\right.} d s .
\end{aligned}
$$

Therefore, unless $F=\Phi$, for small enough $\delta>0$, the right-hand side of the above relation is bounded away from zero for infinitely many $n$. Hence, Theorem A.2 implies that $\frac{1}{\left\|v_{n}\right\|_{2}} y_{n}$ is not normally distributed as $n \rightarrow \infty$.

Proof of Theorem 2: Recall that aggregate volatility is of order $\left\|v_{n}\right\|_{2}$. On the other hand, the fact that all eigenvalues of $(1-\alpha) W_{n}$ lie strictly inside the unit circle means that $v_{n}$ can be expressed in terms of the convergent power series

$$
v_{n}^{\prime}=\frac{\alpha}{n} \mathbf{1}^{\prime} \sum_{k=0}^{\infty}\left[(1-\alpha) W_{n}\right]^{k}
$$

implying that

$$
v_{n}^{\prime} \geq \frac{\alpha}{n} \mathbf{1}^{\prime}+\frac{\alpha(1-\alpha)}{n} \mathbf{1}^{\prime} W_{n}
$$

Therefore,

$$
\begin{aligned}
\left\|v_{n}\right\|_{2}^{2} & \geq \frac{\alpha^{2}}{n^{2}} \mathbf{1}^{\prime} \mathbf{1}+\frac{2 \alpha^{2}(1-\alpha)}{n^{2}} \mathbf{1}^{\prime} W_{n} \mathbf{1}+\frac{\alpha^{2}(1-\alpha)^{2}}{n^{2}}\left\|W_{n}^{\prime} \mathbf{1}\right\|_{2}^{2} \\
& =\frac{\alpha^{2}(3-2 \alpha)}{n}+\frac{\alpha^{2}(1-\alpha)^{2}}{n^{2}}\left\|W_{n}^{\prime} \mathbf{1}\right\|_{2}^{2} \\
& =\Theta(1 / n)+\Theta\left(\frac{1}{n^{2}} \sum_{i=1}^{n} d_{i}^{2}\right)
\end{aligned}
$$

where we have used the fact that the $i$-th column sum of $W_{n}$ is the outdegree of sector $i$, and that the sum of all its elements is equal to $n$. Given that inequality $\sqrt{n}\|z\|_{2} \geq\|z\|_{1}$ holds for any

\footnotetext{
${ }^{28}$ For a similar argument, see Christopeit and Werner (2001).
} 
$n$-dimensional vector $z$, we conclude that

$$
\sum_{i=1}^{n} d_{i}^{2} \geq \frac{1}{n}\left(\sum_{i=1}^{n} d_{i}\right)^{2}=n .
$$

Thus, the first term in (16) is always dominated by the second term. This establishes the first part of the theorem.

To prove the second part of the theorem, note that average outdegree $\bar{d}$ is equal to one. Therefore,

$$
\frac{1}{n^{2}} \sum_{i=1}^{n} d_{i}^{2}=\frac{n-1}{n^{2}}\left[\mathrm{CV}_{n}\right]^{2}+\frac{1}{n}
$$

establishing that $\operatorname{var}\left(y_{n}\right)=\Omega\left(\frac{1+\left(\mathrm{CV}_{n}\right)^{2}}{n}\right)$. This completes the proof.

Proof of Corollary 1: Define

$$
\hat{P}_{n}(k) \equiv \frac{1}{n}\left|\left\{i \in \mathcal{I}_{n}: d_{i}^{2}>k\right\}\right|
$$

as the empirical counter-cumulative distribution function of the outdegrees-squared. By definition, $\hat{P}_{n}(k)=P_{n}(\sqrt{k})$ for all $k$. Also define $B=\left\{b_{1}, \ldots, b_{m}\right\}$ as the set of values that the outdegreessquared of $\mathcal{E}_{n}$ take, where $b_{k+1}>b_{k}$ for all $k$. Thus,

$$
\begin{aligned}
\sum_{i=1}^{n} d_{i}^{2} & =n \sum_{k=1}^{m} b_{k}\left[\hat{P}_{n}\left(b_{k-1}\right)-\hat{P}_{n}\left(b_{k}\right)\right] \\
& =n \sum_{k=0}^{m-1}\left(b_{k+1}-b_{k}\right) \hat{P}_{n}\left(b_{k}\right)
\end{aligned}
$$

with the convention that $b_{0}=0$. Therefore,

$$
\sum_{i=1}^{n} d_{i}^{2}=n \int_{0}^{b_{m}} \hat{P}_{n}(t) d t=2 n \int_{0}^{d_{\max }^{n}} t P_{n}(t) d t
$$

where the last equality is due to a simple change of variables. The assumption that $L(\cdot)$ is a slowlyvarying function, satisfying $\lim _{t \rightarrow \infty} L(t) t^{\delta}=\infty$ for any positive $\epsilon>0$, implies that

$$
\sum_{i=1}^{n} d_{i}^{2} \geq n \hat{c}_{n} \int_{0}^{d_{\max }^{n}} t^{(1-\beta-\delta)} d t
$$

where $\hat{c}_{n}=\Theta(1)$ is a sequence of positive numbers. Thus, from (7) in Theorem 2 and since $\beta \in$ $(1,2)$, we have

$$
\left(\operatorname{var} y_{n}\right)^{1 / 2}=\Omega\left(n^{\frac{1-\beta}{\beta}-\delta^{\prime}}\right),
$$

where $\delta^{\prime}=\delta /(2 \beta)$; completing the proof. 
Proof of Theorem 3: Recall that the influence vector corresponding to economy $\mathcal{E}_{n}$ can be written in terms of a power series of $W_{n}$ specified by (15). Given the fact that all terms in this infinite sum are non-negative vectors, we have

$$
v_{n}^{\prime} \geq \frac{\alpha}{n} \mathbf{1}^{\prime}\left[I+(1-\alpha) W_{n}+(1-\alpha)^{2}\left(W_{n}\right)^{2}\right] .
$$

Therefore,

$$
\begin{aligned}
\left\|v_{n}\right\|_{2}^{2} & \geq \frac{\alpha^{2}}{n^{2}} \mathbf{1}^{\prime}\left[I+(1-\alpha) W_{n}+(1-\alpha)^{2}\left(W_{n}\right)^{2}\right]\left[I+(1-\alpha) W_{n}+(1-\alpha)^{2}\left(W_{n}\right)^{2}\right]^{\prime} \mathbf{1} \\
& =\Theta\left(\frac{1}{n^{2}}\left\|\mathbf{1}^{\prime} W_{n}\right\|_{2}^{2}\right)+\Theta\left(\frac{1}{n^{2}} \mathbf{1}^{\prime}\left(W_{n}\right)^{2} W_{n}^{\prime} \mathbf{1}\right)+\Theta\left(\frac{1}{n^{2}}\left\|\mathbf{1}^{\prime}\left(W_{n}\right)^{2}\right\|_{2}^{2}\right),
\end{aligned}
$$

where we have used the fact that $\frac{1}{n^{2}}\left\|\mathbf{1}^{\prime} W_{n}\right\|_{2}^{2}=\frac{1}{n^{2}} \sum_{i=1}^{n} d_{i}^{2}$ dominates $1 / n$ for large values of $n$. For the second term on the right-hand side of (17), we have

$$
\begin{aligned}
\mathbf{1}^{\prime}\left(W_{n}\right)^{2} W_{n}^{\prime} \mathbf{1} & =\sum_{i=1}^{n} \sum_{j=1}^{n} w_{j i} d_{i} d_{j} \\
& =\sum_{i=1}^{n} \sum_{j \neq i} w_{j i} d_{i} d_{j}+\sum_{i=1}^{n} w_{i i} d_{i}^{2} \\
& =s\left(W_{n}\right)+\mathcal{O}\left(\sum_{i=1}^{n} d_{i}^{2}\right),
\end{aligned}
$$

where $s\left(W_{n}\right) \equiv \sum_{i \neq j} w_{j i} d_{i} d_{j}$ is known as the $s$-metric of the corresponding intersectoral network. On the other hand, for the third term on the right-hand side of (17), we have

$$
\begin{aligned}
\left\|\mathbf{1}^{\prime}\left(W_{n}\right)^{2}\right\|_{2}^{2} & =\sum_{i=1}^{n}\left[\sum_{j=1}^{n} w_{j i} d_{j}\right]^{2}=\sum_{i=1}^{n}\left[w_{i i} d_{i}+\sum_{j \neq i} w_{j i} d_{j}\right]^{2} \\
& =\sum_{i=1}^{n} w_{i i}^{2} d_{i}^{2}+2 \sum_{i=1}^{n} \sum_{j \neq i} w_{i i} w_{j i} d_{i} d_{j}+\sum_{i=1}^{n}\left[\sum_{j \neq i} w_{j i} d_{j}\right]^{2} \\
& =\mathcal{O}\left(\sum_{i=1}^{n} d_{i}^{2}\right)+\mathcal{O}\left(s\left(W_{n}\right)\right)+\sum_{i=1}^{n} \sum_{j \neq i} d_{j}^{2} w_{j i}^{2}+\sum_{i=1}^{n} \sum_{j \neq i} \sum_{k \neq i, j} w_{j i} w_{k i} d_{j} d_{k} \\
& =\mathcal{O}\left(\sum_{i=1}^{n} d_{i}^{2}\right)+\mathcal{O}\left(s\left(W_{n}\right)\right)+\Theta\left(\tau_{2}\left(W_{n}\right)\right)
\end{aligned}
$$

where in the next to the last equality we used the fact that $w_{i i} \leq 1$ for all $i$. The last equality holds because of the fact that $\sum_{i=1}^{n} w_{j i}^{2} \leq 1$ for all $j$. Thus, combining all the above leads to

$$
\left\|v_{n}\right\|_{2}^{2}=\Omega\left(\frac{1}{n^{2}}\left[\sum_{i=1}^{n} d_{i}^{2}+s\left(W_{n}\right)+\tau_{2}\left(W_{n}\right)\right]\right) .
$$


Now, inequality

$$
\sum_{i=1}^{n}\left[d_{i}-\sum_{j \neq i} w_{j i} d_{j}\right]^{2} \geq 0
$$

guarantees that

$$
\sum_{i=1}^{n} d_{i}^{2}+\sum_{i=1}^{n} \sum_{j \neq i} d_{j}^{2} w_{j i}^{2}+\tau_{2}\left(W_{n}\right) \geq 2 s\left(W_{n}\right)
$$

implying that $s\left(W_{n}\right)=\mathcal{O}\left(\sum_{i=1}^{n} d_{i}^{2}+\tau_{2}\left(W_{n}\right)\right)$. Therefore, in highly disaggregated economies, the effect captured by the $s$-metric is dominated by the sum of the other two terms, and as a result

$$
\left\|v_{n}\right\|_{2}=\Omega\left(\frac{1}{n} \sqrt{\sum_{i=1}^{n} d_{i}^{2}}+\frac{\sqrt{\tau_{2}\left(W_{n}\right)}}{n}\right),
$$

completing the proof.

Proof of Corollary 2: By equation (17), we have $\left(\operatorname{var} y_{n}\right)^{1 / 2}=\Omega\left(\frac{1}{n}\left\|\mathbf{1}^{\prime}\left(W_{n}\right)^{2}\right\|_{2}\right)$, which implies that

$$
\left(\operatorname{var} y_{n}\right)^{1 / 2}=\Omega\left(\frac{1}{n} \sqrt{\sum_{i=1}^{n} q_{i}^{2}}\right) .
$$

The rest of the proof follows from an argument similar to the proof of Corollary 1.

Proof of Theorem 4: First, note that $\left\|v_{n}\right\|_{2}=\Omega(1 / \sqrt{n})$ for any sequence of economies. On the other hand, for a balanced sequence of economies, we have $\left\|W_{n}\right\|_{1}=\max _{i \in \mathcal{I}_{n}} d_{i}=\Theta(1)$. Therefore, rearranging equation (4) to

$$
v_{n}^{\prime}=\frac{\alpha}{n} \mathbf{1}^{\prime}+(1-\alpha) v_{n}^{\prime} W_{n}
$$

implies that

$$
\left\|v_{n}\right\|_{\infty} \leq \frac{\alpha}{n}+(1-\alpha)\left\|W_{n}\right\|_{1}\left\|v_{n}\right\|_{\infty} \leq \frac{\alpha}{n}+C(1-\alpha)\left\|v_{n}\right\|_{\infty} .
$$

where $C$ is a constant independent of $n$. Thus, for $\alpha>(C-1) / C$,

$$
\left\|v_{n}\right\|_{\infty} \leq \frac{\alpha}{n}[1-(1-\alpha) C]^{-1},
$$

guaranteeing that $\left\|v_{n}\right\|_{\infty}=\mathcal{O}(1 / n)$. Finally, Hölder's inequality $\left\|v_{n}\right\|_{2} \leq \sqrt{\left\|v_{n}\right\|_{1}\left\|v_{n}\right\|_{\infty}}$ and the fact that $\left\|v_{n}\right\|_{1}=1$ imply that $\left\|v_{n}\right\|_{2}=\mathcal{O}(1 / \sqrt{n})$; completing the proof. 


\section{Appendix D: Higher-Order Interconnections}

As mentioned in Section 3.4, the results on second-order interconnections can be extended even further in order to capture more complex patterns of cascades due to higher-order interconnectivities in the intersectoral network. Mathematically, this will correspond to tighter lower bounds than the one we provided in Theorem 3.

Definition. Given an economy $\mathcal{E}_{n}=\left(\mathcal{I}_{n}, W_{n},\left\{F_{i n}\right\}_{i \in \mathcal{I}_{n}}\right)$, the $(m+1)^{t h}$-order interconnectivity coefficient is defined as

$$
\tau_{m+1}\left(W_{n}\right) \equiv \sum_{i=1}^{n} \sum_{\substack{j_{1}, \ldots, j_{m} \\ k_{1}, \ldots, k_{m} \\ \text { all distinct }}}\left(d_{j_{1}}^{n} d_{k_{1}}^{n}\right)\left(w_{j_{m} i}^{n} w_{k_{m} i}^{n}\right) \prod_{s=1}^{m-1} w_{j_{s} j_{s+1}}^{n} \prod_{r=1}^{m-1} w_{j_{r} j_{r+1}}^{n}
$$

This coefficient captures input-output relations between different sectors of order $m+1$. For example, the third-order coefficient will be high when the suppliers of high-degree sectors share common suppliers. As in the case of second-order interconnectivity coefficient, the Rearrangement Inequality implies that higher levels of $\tau_{m}$ correspond to higher interconnectivities among different sectors. In particular, we have the following generalization of Theorem 3.

Theorem A.3. Consider a sequence of economies $\left\{\mathcal{E}_{n}\right\}_{n \in \mathbb{N}}$. Then for any $m \in \mathbb{N}$, aggregate volatility satisfies

$$
\left(\operatorname{var} y_{n}\right)^{1 / 2}=\Omega\left(\frac{1}{\sqrt{n}}+\frac{\mathrm{CV}_{n}}{\sqrt{n}}+\frac{\sqrt{\tau_{2}\left(W_{n}\right)}}{n}+\cdots+\frac{\sqrt{\tau_{m}\left(W_{n}\right)}}{n}\right) .
$$

The proof follows a logic identical to the proof of Theorem 3. In particular, it is easy to verify that for any positive integer $m$, the influence vector satisfies the following inequality; a consequence of equation (15):

$$
v_{n} \geq \frac{\alpha}{n} \sum_{k=0}^{m}(1-\alpha)^{k} \mathbf{1}^{\prime}\left(W_{n}\right)^{k},
$$

leading to the following lower bound for the Euclidean norm of the influence vector:

$$
\left\|v_{n}\right\|_{2}^{2} \geq \frac{\alpha^{2}}{n^{2}} \sum_{k=1}^{m}(1-\alpha)^{2 k} \mathbf{1}^{\prime}\left(W_{n}\right)^{k}\left(W_{n}^{\prime}\right)^{k} \mathbf{1} .
$$

Writing the matrix powers in terms of the input-output weights, and upon some simplification and rearrangement of terms, we get the result for any positive constant integer $m$. 


\section{References}

Acemoglu, Daron, Asuman Ozdaglar, and Alireza Tahbaz-Salehi (2010), "Cascades in networks and aggregate volatility." Working Paper 16516, National Bureau of Economic Research.

Bak, Per, Kan Chen, José Scheinkman, and Michael Woodford (1993), “Aggregate fluctuations from independent sectoral shocks: self-organized criticality in a model of production and inventory dynamics." Ricerche Economiche, 47, 3-30.

Ballester, Coralio, Antoni Calvó-Armengol, and Yves Zenou (2006), "Who's who in networks. Wanted: The key player." Econometrica, 74, 1403-1417.

Basu, Susanto (1995), "Intermediate goods and business cycles: Implications for productivity and welfare." The American Economic Review, 85, 512-531.

Bonacich, Phil (1987), "Power and centrality: A family of measures." American Journal of Sociology, 92, 1170-1182.

Burress, David (1994), “Homeomorphism between Leontief and Cobb-Douglas input-output models." Economics Letters, 44, 49-53.

Carvalho, Vasco M. (2008), “Aggregate fluctuations and the network structure of intersectoral trade." Working paper.

Carvalho, Vasco M. and Xavier Gabaix (2010), "The great diversication and its undoing." Working Paper 16424, National Bureau of Economic Research.

Christopeit, Norbert and Hans Joachim Werner (2001), "A necessary and sufficient condition for the convergence of a sum of weighting random variables to a normal distribution." Econometric Theory, 17, 857-858.

Ciccone, Antonio (2002), "Input chains and industrialization." Review of Economic Studies, 63, 565587.

Clauset, Aaron, Cosma Rohilla Shalizi, and M. E. J. Newman (2009), "Power-law distributions in empirical data." SIAM Review, 51, 661-703.

Dupor, Bill (1999), "Aggregation and irrelevance in multi-sector models." Journal of Monetary Economics, 43, 391-409.

Durlauf, Steven N. (1993), "Nonergodic economic growth." Review of Economic Studies, 60, 349-366.

Durrett, Rick (2005), Probability: Theory and Examples, third edition. Duxbury Press, Belmont, CA.

Foerster, Andrew T., Pierre-Daniel G. Sarte, and Mark W. Watson (2011), "Sectoral versus aggregate shocks: A structural factor analysis of industrial production." Journal of Political Economy, 119, $1-38$.

Gabaix, Xavier (2011), “The granular origins of aggregate fluctuations." Econometrica, 79, 733-772.

Gabaix, Xavier and Rustam Ibragimov (2011), "Rank-1/2: A simple way to improve the OLS estimation of tail exponents." Journal of Business and Economic Statistics, 29, 24-39. 
Golub, Benjamin and Matthew O. Jackson (2010), "Naïve learning in social networks and the wisdom of crowds." American Economic Journal: Microeconomics, 2, 112-49.

Horvath, Michael (1998), "Cyclicality and sectoral linkages: Aggregate fluctuations from sectoral shocks." Review of Economic Dynamics, 1, 781-808.

Horvath, Michael (2000), "Sectoral shocks and aggregate fluctuations." Journal of Monetary Economics, 45, 69-106.

Hulten, Charles R. (1978), "Growth accounting with intermediate inputs." The Review of Economic Studies, 45, 511-518.

Jackson, Matthew O. (2008), Social and Economic Networks. Princeton University Press, Princeton, NJ.

Jones, Charles I. (2011), "Intermediate goods and weak links: A theory of economic development." American Economic Journal: Macroeconomics, 3, 1-28.

Jones, Charles I. (forthcoming), "Misallocation, economic growth, and input-output economics." In Proceedings of Econometric Society World Congress (Daron Acemoglu, Manuel Arellano, and Eddie Dekel, eds.), Cambridge University Press.

Jovanovic, Boyan (1987), “Micro shocks and aggregate risk." The Quarterly Journal of Economics, $102,395-409$.

Linnik, Yuri V. and Iosif V. Ostrovskiǔ (1977), Decomposition of random variables and vectors, volume 48 of Translations of Mathematical Monographs. American Mathematical Society, Providence, RI. (Translated from Russian).

Long, John B. and Charles I. Plosser (1983), "Real business cycles." Journal of Political Economy, 91, 39-69.

Lucas, Robert E. (1977), “Understanding business cycles." Carnegie-Rochester Conference Series on Public Policy, 5, 7 - 29.

Mulally, Alan R. (2008), "Examining the state of the domestic automobile industry." Hearing, United States Senate Committee on Banking, Housing, and Urban Affairs.

Nadaraya, E. A. (1964), “On estimating regression.” Theory of Probability and its Applications, 9, 141142.

Rotar, Vlademir I. (1975), "An extension of the Lindeberg-Feller theorem." Mathematical Notes, 18, 660-663.

Shea, John (2002), "Complementarities and comovements." Journal of Money, Credit and Banking, $34,412-433$.

Steele, J. Michael (2004), The Cauchy-Schwarz Master Class: An Introduction to the Art of Mathematical Inequalities. Cambridge University Press, New York, NY.

Watson, Geoffrey S. (1964), "Smooth regression analysis." Sankhyā: The Indian Journal of Statistics, Series A, 26, 359-372. 